\title{
Information arms race explains plant-herbivore chemical communication in ecological communities
}

\author{
Pengjuan $\mathrm{Zu}^{1 *}$, Karina Boege ${ }^{2}$, Ek del-Val ${ }^{3}$, Meredith C. Schuman ${ }^{4,5}$, \\ Philip C. Stevenson ${ }^{6,7}$, Alejandro Zaldivar-Riverón ${ }^{8}$, Serguei Saavedra ${ }^{1}$ \\ ${ }^{1}$ Department of Civil and Environmental Engineering, Massachusetts Institute of Technology, \\ 77 Massachusetts Av., 02139 Cambridge, MA, USA \\ ${ }^{2}$ Instituto de Ecología, Universidad Nacional Autónoma de México \\ Av. Universidad 3000, Ciudad Universitaria \\ 04510 Ciudad de México, México \\ ${ }^{3}$ Instituto de Investigaciones en Ecosistemas y Sustentabilidad, \\ Escuela Nacional de Estudios Superiores Unidad Morelia \\ Universidad Nacional Autónoma de México \\ Antigua Carretera a Pátzcuaro 8701, 58190 Morelia, Michoacán, México \\ ${ }^{4}$ Department of Molecular Ecology, Max Planck Institute for Chemical Ecology \\ Hans-Knöll-Strasse 8, Jena DE-07745, Germany \\ ${ }^{5}$ Departments of Chemistry and Geography, University of Zurich \\ Winterthurerstrasse 190, Zurich CH-8057, Switzerland \\ ${ }^{6}$ Royal Botanic Gardens, Kew \\ Richmond, Surrey, TW9 3AB, UK and Natural Resources Institute \\ ${ }^{7}$ University of Greenwich, Chatham, Kent, ME4 4TB, UK \\ ${ }^{8}$ Instituto de Biología, Universidad Nacional Autónoma de México \\ Av. Universidad 3000, Ciudad Universitaria \\ 04510 Ciudad de México, México \\ ${ }^{*}$ Correspondence author
}


1 Plants emit an extraordinary diversity of chemicals, providing information about their identity and mediating interactions with insects. However, most studies have focused on a few model species in controlled environments, limiting our capacity to understand plant-insect chemical communication in ecological communities. Here, by integrating information theory with ecological and evolutionary theories, we show that a stable information structure of plant volatile organic compounds (VOCs) can emerge from a conflicting information process between plants and herbivores. We corroborate this information arms-race theory with field data recording plant-VOC associations and herbivore-plant interactions in a tropical dry forest. We reveal that plant VOC redundancy and herbivore specialization can be explained by a conflicting information transfer. Information-based communication approaches can increase our understanding of species interactions across trophic levels.

One-sentence summary: We find that a stable information structure of plant volatile organic compounds can emerge from a conflicting information process between plants and herbivores in ecological communities. 
Chemical information is an ancient and ubiquitous channel to mediate species interactions (1) (e.g., attracting or repelling individuals) and regarded as one of the main forces shaping plant-herbivore interaction networks (2,3). For example, insects have a large number of olfactory receptors with high sensitivity for chemical signals $(4,5)$, which play essential roles in their fundamental activities (6) including foraging ( 7 ) and oviposition (8). Chemically-mediated species interactions are based on information transfer; which, we define simply as communication regardless of the benefit to the emitter and receiver, following previous work (9). Indeed, understanding the chemical communication between plants and herbivores has been an active field of research (10-12), where the majority of work has focused on how a specific plant (or genus) defends against herbivores directly by using chemical repellents (13)or by producing stronger chemical defenses $(14-16)$, or indirectly by emitting signals to attract herbivores' enemies (e.g., predators or parasitoids) (17-22). So far, only a few descriptive studies (23, 24) have begun to investigate this chemical communication at the community level. More critically, little is known about how information transfer shapes species interactions. For example, it remains unclear how plants code chemical information to deal with a diversity of potential herbivores, and how herbivores decode such olfactory signals to distinguish among plants and identify potential hosts. Even less is known about whether there is any general information structure of plant-herbivore chemical communication, and how such structure can be maintained under an ongoing chemical arms race between plants and herbivores.

Information theory (25), which provides a quantitative and scalable way to measure information transfer, has already brought key insights about the structure and emergence of human language (26) and can be extended to increase our understanding about the "chemical language" in ecological communities (12,27-29). Yet, such attempts have only been employed to study chemical communication of a single plant species with its interacting insects (30). Here, we employ information theory to study plant volatile organic compounds (VOCs) as a communication channel forming plant-insect interaction networks (31). From an information perspective, the relationship between a sender and a receiver (or speaker and hearer) determines the nature of the signal transmission as well as the evolution of the information structure shaping the communication pattern between individuals. That is, individuals can either provide clear information that could be decoded easily or spurious information that could be difficult to decode. Moreover, a conflicting or harmonious communication process between sender and receiver can be 
modeled by the combinations of maximizing or minimizing uncertainty (or mutual information) of the two parties, respectively (26).

Specifically, for a given communication system that is formalized by a matrix describing how a vector of signals $(\mathrm{S})$ is associated with a vector of objects $(\mathrm{O})$, the clearness (or the spuriousness) of the communication can be described by two important information measurements (25): First, mutual information $(I(O, S))$ describes the amount of information that one can obtain about objects by knowing the signals. Second, conditional entropy $(H(O \mid S))$ describes the uncertainty of correctly identifying an object given that a specific signal has been observed (Ref. (32) provides a detailed mathematical account of these expressions). Importantly, both mutual information and conditional entropy represent the efficiency of the coding (and decoding) strategies and can be related to the fitness of senders and receivers (26).

Tropical dry forests host a large number of interacting and co-evolving plant and butterfly species and are regarded as biodiversity hotspots (33). Our field work was conducted in a tropical dry forest of the Chamela-Cuixmala Biosphere Reserve $\left(19^{\circ} 22^{\prime}-19^{\circ} 39^{\prime} \mathrm{N}, 104^{\circ} 56^{\prime}-105^{\circ} 10^{\prime} \mathrm{W}\right)$ in Jalisco, Mexico. During the rainy season of 2018, we searched comprehensively for Lepidopteran larvae on leaves of target plants in our transect plots and reared the larvae in the laboratory with leaves collected from their host plant species to confirm their trophic interaction and identify the herbivore species. Ref. (32) provides a detailed account of plants and insects, sampling, and identification procedures. We constructed a qualitative (presence/absence) animal-plant $(A P)$ interaction matrix comprising 28 Lepidopteran herbivore species and 20 plant species. The $A P$ matrix was rather sparse (see Figure 1A, Table S1) with a median of 1 plant species per herbivore (ranging from 1 to 9 plant species per herbivore).

Additionally, we sampled the headspace around leaves to retrieve VOCs from each of these plant species. We were able to match 93 analytes from headspace samples to the NIST17 VOC library, of which 56 were likely biogenic (from plants, or from plants and microbes), and 31 of these biogenic VOCs could be identified by one or more abundant ions in an untargeted analysis. We used single representative ions from these 31 VOCs for quantification. Ref. (32) provides a detailed account of VOC sampling, identification, and quantification procedures. Figure 1B (and Table S2) shows that, contrary to the $A P$ matrix, the plant-VOC $(P V)$ association matrix is dense and erratic with a median of 28 VOCs per plant (ranging from 23 to 30 VOCs ). 
Importantly, the $P V$ matrix provides information about plant-VOC associations, whereas the $A V$ matrix (mathematically defined by the matrix multiplication $A P \times P V$ ) provides information about herbivore-VOC associations. These associations describe the patterns of plants emitting information and herbivores receiving it, and thus represent plant coding and herbivore decoding strategies. The $A V$ matrix (Fig. 1C) is dense and weighted, indicating that individual VOCs are associated with more than one herbivore. Note that to generate the herbivore-VOC association ( $A V$ matrix) we assumed that if a herbivore can feed on a plant, it must be able to cope with spatial and temporal variation in VOCs that are used to distinguish suitable hosts, despite the effects of these VOCs on the herbivore (attract, deter, neutral). Altering either the $P V$ or $A P$ matrix can change the herbivore-VOC associations ( $A V$ matrix).

We built the conceptual framework for our study based on a conflicting optimization process between plants and herbivores (see Fig. 2). Specifically, we hypothesized that plants aim to decrease the decoding efficiency of herbivores via changing plant-VOC associations (PV), whereas herbivores aim to increase this efficiency via changing herbivore-plant interactions (AP). Ecologically, the decoding strategy can be linked to the fitness of plants and herbivores. A high decoding efficiency can increase the attack rates and decrease the fitness of plants. In contrast, low efficiency can increase the searching time and decrease the fitness of herbivores (12). Formally, we define the fitness relationships as $\mathrm{F}_{P}=H(A \mid V) \in[0,1]$ and $\mathrm{F}_{A}=1-H(V \mid A) \in[0,1]$, respectively. That is, plant fitness is proportional to the conditional entropy (uncertainty) between VOCs and herbivores, whereas herbivore fitness is negatively proportional to the conditional entropy between herbivores and VOCs. Ref. (32) provides details on the calculations.

To track the evolutionary trajectory of the communication system between plants and herbivores based on our hypothesis, we simulated the optimization process above in the following way. Plants and herbivores can increase their fitness relationships (based on the $A V$ matrix) via modifying the interactions in the $P V$ matrix and in the $A P$ matrix, respectively (see Figure 2). These modifications come from random mutations of a given number of elements in the $P V$ and $A P$ matrices. We assumed equal mutation rate for each link (plant-VOC link or herbivore-plant link) in the absence of any prior knowledge. Therefore, the number of random mutations that plants and herbivores can have for each round were proportional to the number of all possible links (i.e., number of elements in $P V$ and $A P$ matrices). Mutations are only selected if they increase the corresponding fitness relationships. To mimic a continuous arms race, we only allowed changes by 
plants and herbivores in an alternating fashion (see Figure 2). Both the $P V$ and $A P$ matrices can be initialized from any random configuration with the only restriction of matrix size. To make our simulation as simple as possible, we assumed that plants can potentially emit any VOC and herbivores can potentially eat any plant without any phylogenetic or physiological constraints.

We investigated the capacity of our hypothesized mechanism to explain both the (de)coding strategies and the information structure of chemical communication between plants and herbivores observed in the field. Specifically, we determined the ability of our model to explain the conditional entropies $H(P \mid V), H(A \mid P)$, and $H(A \mid V)$; which characterize the $P V, A P$, and $A V$ matrices, respectively. Then, we determined its ability to explain the observed fitness relationships $F_{P}$ and $F_{A}$. Note that conditional entropy by definition is the average entropy given each specific signal (i.e., individual VOC in the case of $H(P \mid V)$ and $H(A \mid V)$ ). Furthermore, we tested how accurately our model could generate the cumulative information structure given a combination of VOCs by calculating the mutual information between plants and VOCs $(I(P, V))$ and between herbivores and VOCs $(I(A, V))$ as a function of the number of VOCs.

Additionally, we compared our proposed optimization mechanism against the three following alternative optimization mechanisms: The fitness relationships of plants and herbivores depend on their capacity to (1) maximize uncertainty (i.e., $\mathrm{F}_{P}=H(A \mid V)$ and $\mathrm{F}_{A}=H(V \mid A)$ ), (2) minimize uncertainty (i.e., $\mathrm{F}_{P}=1-H(A \mid V)$ and $\mathrm{F}_{A}=1-H(V \mid A)$ ), and (3) minimize and maximize uncertainty (i.e., $\mathrm{F}_{P}=1-H(A \mid V)$ and $\mathrm{F}_{A}=H(V \mid A)$ ). We also ran all our simulations over different initial conditions, ranging from highly specialized to highly generalized matrices. The rationale for using these alternative mechanisms and initializations is to illustrate that potential matches between our theoretical expectations and observed values are not just an artifact of sample size, metrics, any given optimization process, or the high (low) connectivity observed in the PV (AP) matrix. Ref. (32) provides all the details about these additional analyses.

Because sampling bias is an important concern in the majority of ecological studies (34), we tested whether our results would be affected by the sampling effort in reporting species, by an incomplete plant VOC profile, and by sampling the interaction network in a different year or place. We found that all our results were qualitatively equivalent when using sub-samples from the whole dataset (illustrating the scalability of our findings), changing the VOC profile from 31 to 52 by including potential undefined biogenic VOCs, and using information of plant-herbivore interaction networks from previous years (from 2007-2017) (35). Ref. (32) provides all the details 
about the robustness of our results to changes in community size, number of VOCs, and identity and number of species interactions.

Figure 3 shows that the proposed, conflicting, information process is able to explain the coding and decoding patterns observed in the field. In particular, Figure 3A shows that the conditional entropies $H(P \mid V)$ (see triangles), $H(A \mid P)$ (see squares), and $H(A \mid V)$ (see circles) remain bounded across the simulated time to values close to those observed (see dashed, dotted and solid lines). Similarly, Figure 3B shows that the optimized fitness relationships of plants $F_{P}$ (see + symbols) and herbivores $F_{A}$ (see $\times$ symbols) quickly converge to a stable value close to that observed (see solid lines). Importantly, Figure 3C shows that the alternative optimization mechanisms are not able to explain all the patterns.

Additionally, Figure 4 shows that of our four tested models, only our proposed conflicting optimization process can recover the information structure observed in the field. Recall that we measured the cumulative information structure by the mutual information $(I(P, V)$ and $I(A, V))$, where values closer to one imply that the presence or absence of a VOC (or a combination of VOCs) gives more information or has higher probability to tell all the species apart. In fact, the field data revealed that as few as 8 VOCs can tell all the plants apart and 15 VOCs can give $97 \%$ information about herbivores (i.e., have $97 \%$ of probability to correctly tell all the herbivores apart). Importantly, this structure was closely recovered by our proposed optimization mechanism (9 VOCs yield all the information of plants, and 14 VOCs for $97 \%$ information of herbivores, red lines). In contrast, the three alternative optimization mechanisms yield very different information structures, where either a large number of VOCs would be needed to uniquely identify species or the identification from VOCs would be less likely. For example, in the case where both parties aim to maximize uncertainty (i.e., confuse their opponents as in a mutually competitive relationship, blue lines), even by using the combination of all VOCs, it would be impossible to tell all the species apart. All these results are robust to changes in community size, number of VOCS, the identity and number of species interactions, and different initial conditions (see Ref. (32) Fig. S1-S6).

While a few recent studies have begun to demonstrate chemical patterns associated with plant-insect communities $(24,31,36)$, our study proposes a plausible theoretical framework that explains and recovers patterns of information transfer between plants and herbivores from empirical data. Our work is hypothesis-driven and suggests that an information arms race 
between plants and herbivores can drive plants to produce VOCs that are commonly shared by other species, increasing the difficulty for herbivores to identify suitable plants and potentially pressuring herbivores to specialize on a few plants (as the AP matrix shows in Fig. 1). Indeed, previous studies have shown a substantial overlap among the VOCs emitted by different plant species $(31,37,38)$. However, functional roles of a large portion of plant VOCs have been unexplained and overlooked because most mechanistic studies have focused on specific plant species and on the ecological functions of only a few VOCs (12). Here, our information arms-race theory suggests that these seemingly redundant VOCs play an important role in confusing herbivores at the community level. Focusing on herbivore-plant interactions, herbivores are commonly regarded as specialists (6), which was also recovered from our theory. In fact, it is estimated that $<10 \%$ of all herbivores feed on plants that span more than 3 families (39). Our findings further suggest that a conflicting information process drives the rapid accumulation of information by adding VOCs (Fig. 4), contrary to other optimization processes. This provides additional evidence for an arms-race process explaining the large diversity of VOCs in nature, where herbivores have the evolutionary potential to quickly tell all plant species apart by making use of the few most informative VOCs and plants can in-turn respond to this potential by adding more VOCs to their profile. Interestingly, under the same process, herbivores themselves can also be identified using a set of informative VOCs (Fig. 4B). This raises the question of how these VOC profiles result in evolutionary trade-offs regulating the attraction of herbivores and their predators and parasitoids $(17,18)$. Overall, our study suggests information-transfer processes are key drivers of the formation and maintenance of species interactions across trophic levels. 


\section{REFERENCES AND NOTES}

1. Bradbury JW, Vehrencamp SL, et al. (1998) Principles of animal communication (Sinauer Associates).

2. Ehrlich PR, Raven PH (1964) Butterflies and plants: a study in coevolution. Evolution 18:586-608.

3. Richards LA, et al. (2015) Phytochemical diversity drives plant-insect community diversity. Proceedings of the National Academy of Sciences 112:10973-10978.

4. Kaupp UB (2010) Olfactory signalling in vertebrates and insects: differences and commonalities. Nature Reviews Neuroscience 11:188.

5. Hansson BS, Stensmyr MC (2011) Evolution of insect olfaction. Neuron 72:698-711.

6. Schoonhoven LM, Van Loon B, van Loon JJ, Dicke M (2005) Insect-plant biology (Oxford University Press on Demand).

7. Schiestl FP (2010) The evolution of floral scent and insect chemical communication. Ecology Letters 13:643-656.

8. Renwick J, Chew F (1994) Oviposition behavior in lepidoptera. Annual Review of Entomology 39:377-400.

9. Karban R (2008) Plant behaviour and communication. Ecology Letters 11:727-739.

10. Bruce TJ, Pickett JA (2011) Perception of plant volatile blends by herbivorous insects-finding the right mix. Phytochemistry 72:1605-1611.

11. Schuman MC, Baldwin IT (2016) The layers of plant responses to insect herbivores. Annual Review of Entomology 61:373-394.

12. Kessler A, Kalske A (2018) Plant secondary metabolite diversity and species interactions. Annual Review of Ecology, Evolution, and Systematics 49:115-138.

13. Duffey SS, Stout MJ (1996) Antinutritive and toxic components of plant defense against insects. Archives of Insect Biochemistry and Physiology: Published in Collaboration with the Entomological Society of America 32:3-37. 
14. Helms AM, De Moraes CM, Tooker JF, Mescher MC (2013) Exposure of solidago altissima plants to volatile emissions of an insect antagonist (eurosta solidaginis) deters subsequent herbivory. Proceedings of the National Academy of Sciences 110:199-204.

15. Bittner N, Hundacker J, Achotegui-Castells A, Anderbrant O, Hilker M (2019) Defense of scots pine against sawfly eggs (diprion pini) is primed by exposure to sawfly sex pheromones. Proceedings of the National Academy of Sciences 116:24668-24675.

16. Magalhães DM, Da Silva ITFA, Borges M, Laumann RA, Blassioli-Moraes MC (2019) Anthonomus grandis aggregation pheromone induces cotton indirect defence and attracts the parasitic wasp bracon vulgaris. Journal of experimental botany 70:1891-1901.

17. Turlings TC, Erb M (2018) Tritrophic interactions mediated by herbivore-induced plant volatiles: mechanisms, ecological relevance, and application potential. Annual Review of Entomology 63:433-452.

18. De Moraes CM, Lewis W, Pare P, Alborn H, Tumlinson J (1998) Herbivore-infested plants selectively attract parasitoids. Nature 393:570.

19. Poelman EH, Dicke M (2018) Plant-mediated interactions among insects within a community ecological perspective. Annual Plant Reviews online pp 309-337.

20. Kessler A, Baldwin IT (2001) Defensive function of herbivore-induced plant volatile emissions in nature. Science 291:2141-2144.

21. Kessler A, Heil M (2011) The multiple faces of indirect defences and their agents of natural selection. Functional Ecology 25:348-357.

22. Becerra JX, Noge K, Venable DL (2009) Macroevolutionary chemical escalation in an ancient plant-herbivore arms race. Proceedings of the National Academy of Sciences 106:18062-18066.

23. Kantsa A, et al. (2017) Community-wide integration of floral colour and scent in a mediterranean scrubland. Nature Ecology \& Evolution 1:1502.

24. Filella I, et al. (2013) Floral advertisement scent in a changing plant-pollinators market. Scientific Reports 3:3434.

25. Shannon CE (1948) A mathematical theory of communication. Bell System Technical Journal $27: 379-423$. 
26. Ferrer-i Cancho R, Solé RV (2003) Least effort and the origins of scaling in human language. Proceedings of the National Academy of Sciences 100:788-791.

27. Margalef R (1968) Perspectives in Ecological Theory (University of Chicago Press, Chicago).

28. Ulanowicz RE (2001) Information theory in ecology. Computers E3 Chemistry 25:393-399.

29. O'Connor MI, et al. (2019) Principles of ecology revisited: integrating information and ecological theories for a more unified science. Frontiers in Ecology and Evolution 7:219.

30. Doyle L (2009) Quantification of information in a one-way plant-to-animal communication system. Entropy 11:431-442.

31. Vivaldo G, Masi E, Taiti C, Caldarelli G, Mancuso S (2017) The network of plants volatile organic compounds. Scientific reports 7:1-18.

32. See the supplementary material.

33. Miles L, et al. (2006) A global overview of the conservation status of tropical dry forests. Journal of Biogeography 33:491-505.

34. Chase JM, Knight TM (2013) Scale-dependent effect sizes of ecological drivers on biodiversity: why standardised sampling is not enough. Ecology Letters 16:17-26.

35. Boege K, et al. (2019) Temporal variation in the influence of forest succession on caterpillar communities: A long-term study in a tropical dry forest. Biotropica 51:529-537.

36. Kantsa A, Raguso RA, Lekkas T, Kalantzi OI, Petanidou T (2019) Floral volatiles and visitors: A meta-network of associations in a natural community. Journal of Ecology $107: 2574-2586$.

37. Courtois EA, et al. (2009) Diversity of the volatile organic compounds emitted by 55 species of tropical trees: a survey in french guiana. Journal of Chemical Ecology 35:1349-1342.

38. Knudsen JT, Eriksson R, Gershenzon J, Ståhl B (2006) Diversity and distribution of floral scent. The Botanical Review 72:1.

39. Bernays E, Graham M (1988) On the evolution of host specificity in phytophagous arthropods. Ecology 69:886-892. 
40. Hebert PDN, Stoeckle MY, Zemlak TS, Francis CM (2004) Identification of birds through dna barcodes. PLoS Biology 2:e312.

41. Kallenbach M, Veit D, Eilers EJ, Schuman MC (2015) Application of silicone tubing for robust, simple, high-throughput, and time-resolved analysis of plant volatiles in field experiments. Bio-protocol 5(3):e1391.

42. R Core Team (2017) R: A Language and Environment for Statistical Computing ( R Foundation for Statistical Computing, Vienna, Austria).

43. Qi J, et al. (2016) Oral secretions from mythimna separata insects specifically induce defence responses in maize as revealed by high-dimensional biological data. Plant, cell $\mathcal{E}$ environment 39:1749-1766.

44. Villa-Galaviz E, Boege K, del Val E (2012) Resilience in plant-herbivore networks during secondary succession. PLOS ONE 7:e53009.

45. Saavedra S, et al. (2017) A structural approach for understanding multispecies coexistence. Ecological Monographs 87:470-486. 


\section{ACKNOWLEDGEMENTS}

We thank Inari Sosa Aranda and Richard Moore for their help in field work, Geoffey Kite for his help with GC-MS, and Rubén Pérez-Ishiwara for logistic support. We thank Rayko Halitschke at the Max Planck Institute for Chemical Ecology for providing silicon tubing for volatile sampling. We thank Mohammad AlAdwani, Simone Cenci, Lucas Medeiros, and Chuliang Song for numerous constructive discussions about this work. We thank Richard Karban and Robert Ulanowicz for their valuable comments on the manuscript. Funding: The project was funded by grant to PZ from Swiss National Science Foundation number P2ZHP3_178087. EDV and KB are grateful to PAPIIT-UNAM (IN211916) for field work funding. SS acknowledges support from the Mitsui Chair. MCS acknowledges support from the Max Planck Society and the University of Zurich Research Priority Program on Global Change and Biodiversity. Author contributions: PZ and SS designed the study. PZ and EdV collected field data. KB, EdV and AZR identified herbivores and constructed plant-herbivore networks, PZ, MCS and PCS performed chemical analyses. PZ and SS performed theoretical analyses. The manuscript was written primarily by PZ and SS with contributions by MCS and all other co-authors. Competing interests: The authors declare no competing interests. Data and materials availability: All source data and $\mathrm{R}$ code needed to reproduce the results are publicly available in Ref. (32) and at https://github.com/MITEcology/Science_Zu_et_al_2020 (DOI: https://zenodo.org/badge/latestdoi/250857831). 


\section{Figure legends}

Fig. 1. Field data on herbivore (animal)-plant interactions, plant-VOC associations, and herbivore-VOC associations. Panel A, each row and column represents one of the 28 insect herbivores and 20 plants sampled, respectively. Black and white squares show that a given herbivore was, or was not observed feeding on a given plant, respectively. We refer to this as the $A P$ matrix and we use it to investigate the plant-herbivore interaction network. Panel $\mathbf{B}$, each row and column represent one of the 20 plants and 31 VOCs sampled, respectively. Black and white squares show that a given VOC was present or absent in a given plant, respectively. We refer to this as the $P V$ matrix and we use it to investigate the coding strategy of plants. Panel $\mathbf{C}$ shows the matrix of herbivore-VOC associations produced by the multiplication of the previous two matrices $(A P \times P V)$. Each row and column represents one of the 28 herbivores and 31 VOCs associated, respectively. The darker the squares, the greater the frequency with which a given herbivore is associated with a given VOC. We refer to this as the $A V$ matrix and we use it to investigate the decoding strategy of herbivores. See Ref. (32) Table S1-S2 for more information about these matrices.

\section{Fig. 2. Conceptual diagram of the proposed, conflicting, information process} (information arms race) between plants and insect herbivores. The coding strategy by plants is characterized by the plant-VOC $(P V)$ association matrix (green right box). The animal-plant $(A P)$ interaction matrix (blue left box) is used to infer how plants' codes are decoded by herbivores. These matrices undergo mutations by changing any of their elements (e.g., red numbers): zeros and ones correspond to absence and presence, respectively. These mutations will fixate only if the fitness relationship of the corresponding species increases. The fitness relationship of species is defined by herbivores' decoding efficiency based in the communication system, i.e., the $A V$ matrix that results from the product of $A P$ and $P V$ matrices. Specifically, we defined plant and herbivore fitness relationships by the expressions $F_{P}=H(A \mid V)$ and $F_{A}=1-H(V \mid A)$, where $H(\cdot)$ corresponds to the conditional entropy. To increase this fitness relationship, plants and herbivores go through an alternating optimization process, mimicking their arms race. Main text and Ref. (32) provide details about this process. 
Fig. 3. Simulated results based on the information arms-race theory recover the observed information structures in the field. Panel A illustrates the simulation over 1000 time steps derived from our proposed optimization mechanism (information arms-race). The simulation is randomly initialized. The circles, triangles and squares correspond to the conditional entropies $H(A \mid V), H(P \mid V)$ and $H(A \mid P)$, respectively. The solid, dotted and dashed lines stand for the corresponding conditional entropies from field observations. For the same simulation, in Panel $\mathbf{B}$, the + and $\times$ symbols correspond to the fitness relationships of plants $\left(F_{P}\right)$ and herbivores $\left(F_{A}\right)$, respectively. The solid lines correspond to the field observations. Panel $\mathbf{C}$ shows the summary of the observed and simulated values (mean \pm s.d.) based on the last 250 steps (the rectangle window in Panel A) when reaching equilibrium for our proposed mechanism (in red; plants aim to maximize, $\max _{A}$, and herbivores aim to minimize, $\min _{P}$, uncertainty of information, see Fig. 2) and the corresponding ones for the three alternative mechanisms (in green, yellow, and blue; the alternative combinations of the maximization and minimization).

Fig. 4. Emergence of cumulative mutual information. The figure shows the information structure characterized by the cumulative mutual information of $\mathbf{A}$ ) plants and VOCs, i.e., $I(P, V)$ and that of $\mathbf{B})$ herbivores and VOCs, i.e., $I(A, V)$. The higher the value, the better that the presence or absence of VOCs can identify plants. The cumulative mutual information is calculated as a function of the number of VOCs. The black curve corresponds to the field observation, whereas the colored curves correspond to the proposed (in red) and alternative optimization mechanisms. Dashed lines correspond to the minimum number of VOCs that gives the best mutual information according to the field data. 
${ }_{354}$ Supplementary Materials

355 science.sciencemag.org/content/XXX

356 Materials and Methods

357 Supplementary Text

358 Figs. S1-S6

359 Tables S1-S2

360 Databases S1

$361 \quad \mathrm{R}$ scripts as zipped archives

362 References (40-45) 


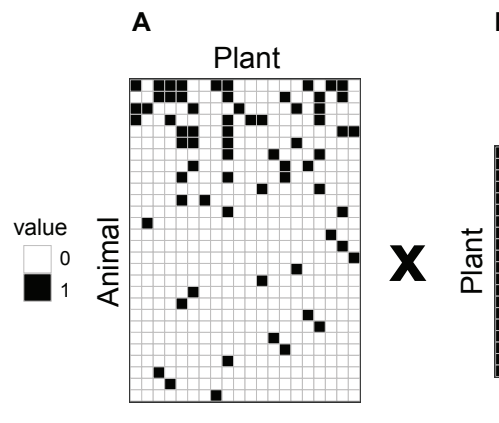

B

C

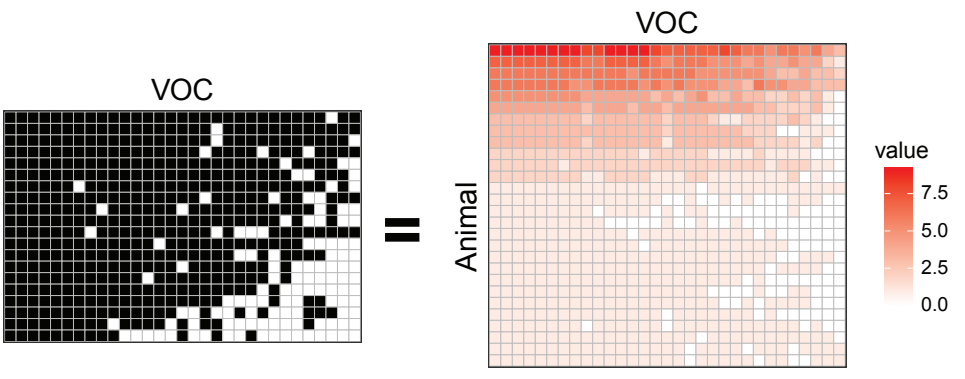

Fig. 1 


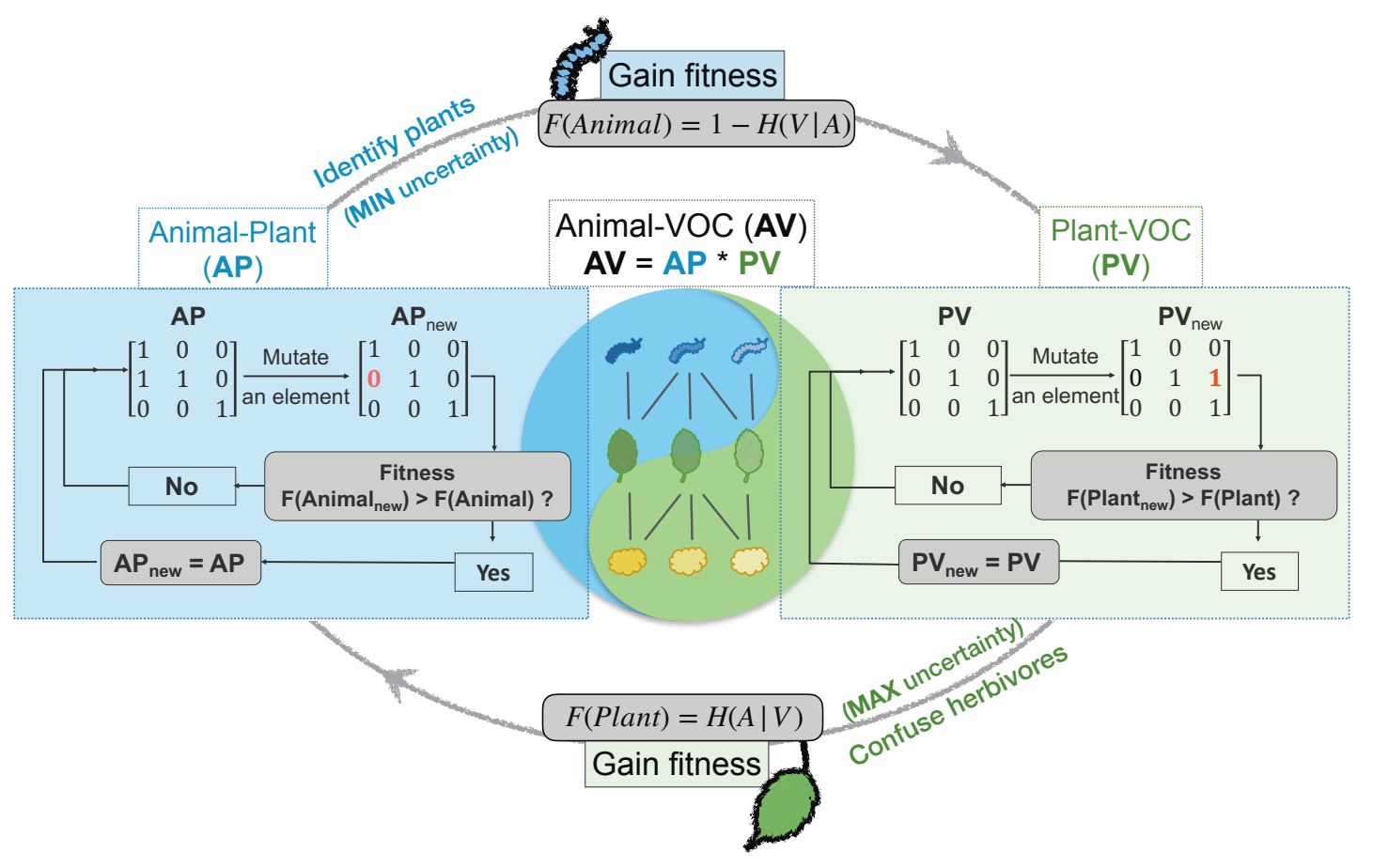

Fig. 2 

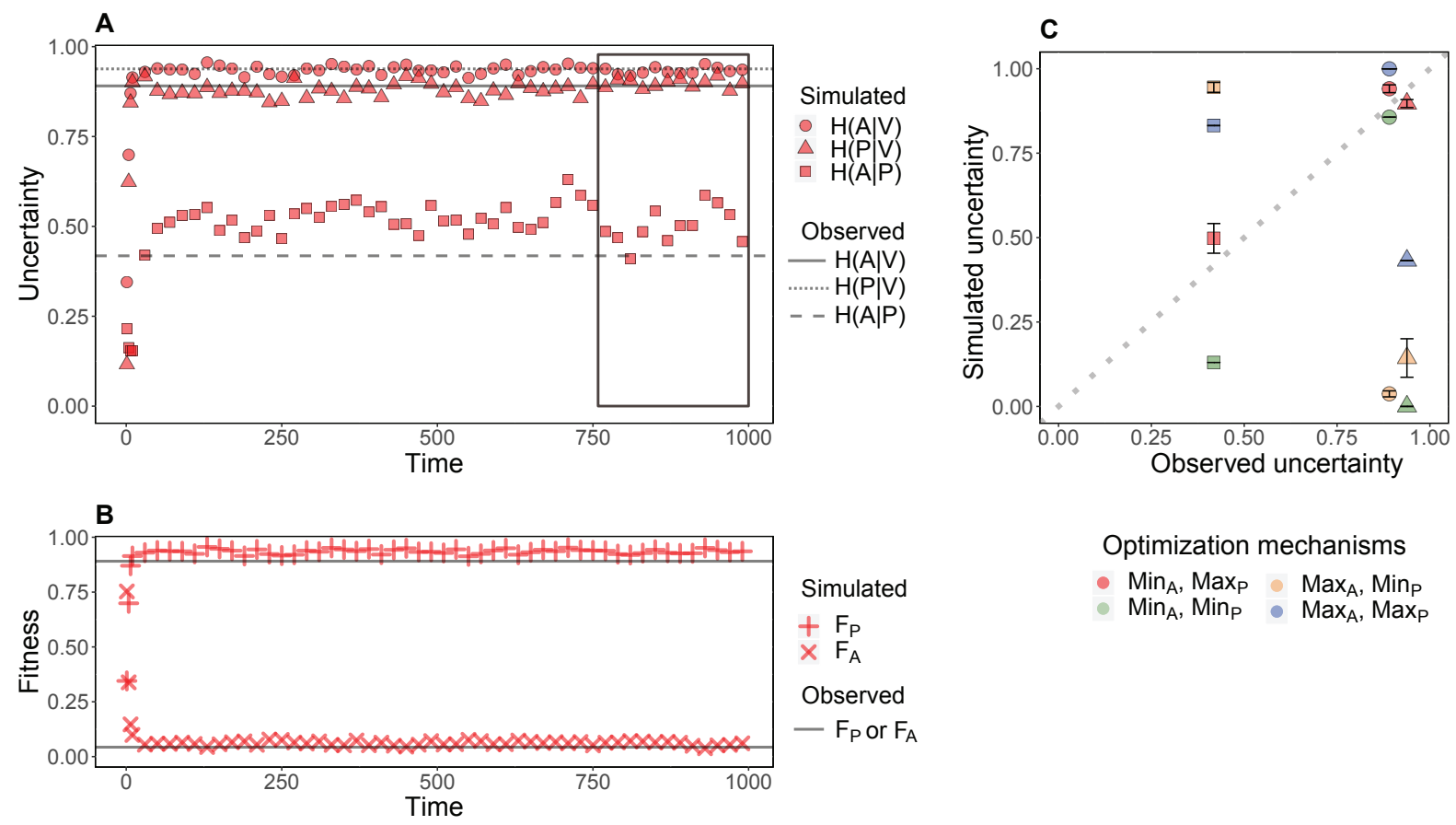

Optimization mechanisms

- $\operatorname{Min}_{A}, \operatorname{Max}_{P} \circ \operatorname{Max}_{A}, \operatorname{Min}_{P}$

- $\operatorname{Min}_{\mathrm{A}}, \operatorname{Min}_{\mathrm{P}} \bullet \operatorname{Max}_{\mathrm{A}}, \operatorname{Max}_{\mathrm{P}}$

Fig. 3 

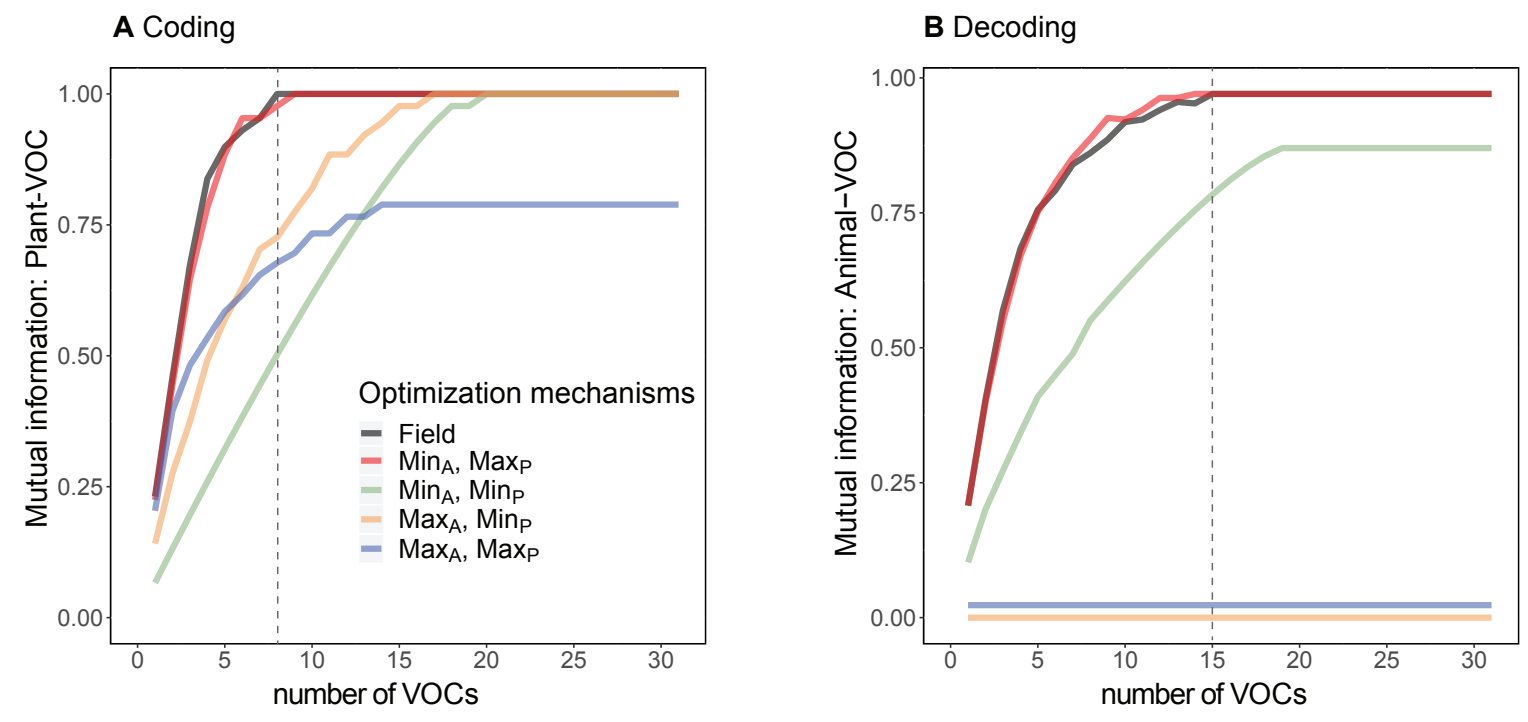

Fig. 4 


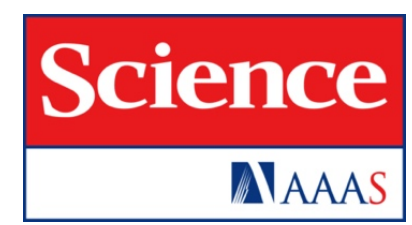

\section{Supplementary Materials for}

Information Arms Race Explains Plant-herbivore Chemical Communication in Ecological Communities

Pengjuan $\mathrm{Zu}^{1 *}$, Karina Boege ${ }^{2}$, Ek del-Val ${ }^{3}$, Meredith C. Schuman ${ }^{4,5}$, Philip C. Stevenson ${ }^{6,7}$, Alejandro Zaldivar-Riverón ${ }^{8}$, Serguei Saavedra ${ }^{1}$

correspondence to: Pengjuan.zu@gmail.com

This PDF file includes:

Materials and Methods
A. Field data
B. Information theory
C. Simulation and statistics
D. Robustness tests concerning effects of sampling bias

Figs. S1 to S6

Tables S1 to S2

References

\section{Other Supplementary Material for this manuscript includes the following:}

Databases S1: Zu_etal_2020_Data_Archive.xlsx

R scripts as zipped archives: $\bar{Z} u$ uetal_2020_R_example.zip

(https://github.com/MITEcology/Science_Zu et_al_2020) 


\section{Materials and Method}

\section{A Field data}

\section{Field site}

The field data were collected in 2018 from a tropical dry forest located in the surroundings Chamela-Cuixmala Biosphere Reserve (19 $\left.22^{\prime}-19^{\circ} 39^{\prime} \mathrm{N}, 104^{\circ} 56^{\prime}-105^{\circ} 10^{\prime} \mathrm{W}\right)$ in Jalisco, Mexico. This site has been established for plant-herbivore network annual surveys during rainy seasons since 2007. Annual average precipitation during the past 12 years ranged from 523 to $1172 \mathrm{~mm}$. There are three stages of secondary succession of the forest: mature (more than 50 years without anthropogenic perturbations), intermediate (approximately 20 years after being excluded and protected from cattle ranching use), and early (approximately 6 years after being excluded and protected). We targeted 20 tree species in plots belonging to the three stages (See Tables S1-S2 for a detailed list of species).

\section{Construction of field plant-herbivore network}

To sample herbivores within each plot, we established four transects of $2 \times 20 \mathrm{~m}$ every $10 \mathrm{~m}$, in which we marked all the target tree species with diameter $\geq 1 \mathrm{~cm}$ and height $\geq 50 \mathrm{~cm}$. We searched for Lepidopteran larvae on all the leaves up to 3 meters of the target trees, and in the cases of taller trees, we randomly chose 3 branches per individual for searching. We conducted a monthly census during the four-month rainy season. We reared larvae to adulthood in the laboratory with leaves from the species on which they were found to confirm their trophic interaction. Lepidopterans were identified using traditional taxonomy complemented with molecular identification (we used the standard DNA barcoding for insects: cytochrome oxidase I, or COI, mitochondrial DNA) of operational taxonomic units $(35,40)$, especially for those cases in which adults failed to emerge. We edited sequences with Sequencher version 4.0.5 (Gene Codes) and aligned them manually based on their translated amino acids. All caterpillars and their host plants registered within the year in each plot were considered as a pooled community, hence we had a total of 29 herbivore species on the targeted plant species. Specific details on the sampling design, molecular identification and species identities can be found in Ref. (35)

\section{Plant VOC collection and construction of plant-VOC network}

We sampled the leaf headspace from three individuals each of the 20 target tree species that were sampled in the field from August $27^{\text {th }}-31^{\text {st }}, 2018$. We used polydimethysiloxane (PDMS) tubing (provided by the Max Planck Institute for Chemical Ecology, Jena, Germany) following the method developed in Ref. (41). Specifically, we selected leaves with no or minimal damage and gently covered it with a cleaned Polyethylene tetraphthalate (PET) cup. A PET lid was also used to close the cup, with a hole allowing the petiole to penetrate. We punctured two holes (one at the bottom of the cup, one in the center of lid where the petiole went through) to avoid moisture accumulation. We placed five pieces of silicone tubing (each $5 \mathrm{~mm}$ length, $1 \mathrm{~mm}$ i.d. $\times 1.8 \mathrm{~mm}$ o.d.) in each cup and left these to equilibrate for 24 hours to avoid differences due primarily to daily dynamics of VOC emission. We set two to three air controls from empty cups for each successional stage plot and each round of VOC sampling. At the end of each sampling, 
we removed the leaves from the cups and transferred the silicone tubing from each sample into a $1.5 \mathrm{ml}$ glass vial (Sigma-Aldrich Chemie Gmbh, Munich, Germany), and screwed on the vial lid (with PTFE/silicone septum bonded, Sigma-Aldrich Chemie Gmbh Munich, Germany). We stored samples in a $-20^{\circ} \mathrm{C}$ freezer before transport to the Royal Botanic Gardens, Kew, London, UK for analysis.

To analyze the leaf VOC samples, we used an Agilent Technologies (Palo Alto, CA, USA, 7890A) gas chromatograph connected to an Agilent Technologies 5975C MSD mass spectrometer (GCMS) coupled with a Markes International TD100 thermal desorption auto-sampler. We placed two silicone tubes from each sample into the sample tray and injected desorbed volatiles into the GC under a temperature program following Ref. (41). The GC was equipped with an Agilent Technolgies DB-5 MS capillary column (30m length, $0.25 \mathrm{~mm}$ diameter, $0.25 \mu \mathrm{m}$ film thickness). We set the temperature of the $\mathrm{GC}$ oven to $40^{\circ} \mathrm{C}$ ( $5 \mathrm{~min}$ hold) and increased to $185^{\circ} \mathrm{C}$ at $5^{\circ} \mathrm{C} / \mathrm{min}$, then increased to $280^{\circ} \mathrm{C}$ at $30^{\circ} \mathrm{C} / \mathrm{min}(0.83 \mathrm{~min}$ hold $)$. We used helium as the carrier gas, with a constant flow of $40 \mathrm{~cm} /$ second.

To retrieve leaf VOC profiles, we used an untargeted analysis to screen all the peaks across all the samples in R (42)using the package XCMS as described in Qi et al. 2016 (43). We removed the typical contamination peaks, and then compared the analytes from headspace samples with the National Institute of Standards and Technologies NIST17 mass spectral library. The relative retention of different identified compounds was checked against that expected for a DB5 column. This allowed the assignment of 93 analytes (although did not distinguish enantiomers where they may have occurred e.g., (+) and (-) $\alpha$-pinene) of which 56 were likely biogenic (from plants, or from plants and microbes), and 31 of the biogenic VOCs could be identified by one or more abundant ions (i.e., ions specific to the analyte and having at least $20 \%$ of the maximum relative intensity across the electron impact mass spectrum) in an untargeted analysis. Each VOC may be represented by a "fingerprint" of multiple $\mathrm{m} / \mathrm{z}$ values. We chose one $\mathrm{m} / \mathrm{z}$ value to represent one VOC. If we had used all $\mathrm{m} / \mathrm{z}$ values for each VOC, this would have produced multiple peaks per VOC (redundant information) as well as different numbers of peaks for different VOCs. An alternative is to use the TIC (total ion chromatogram) but that is less specific and is inaccurate in cases where there is chromatographic overlap of VOCs, or of VOCs with contaminants (41). Then we generated qualitative data ( 1 for presence, 0 for absence) for each species by comparing the peaks in the samples to those in the air controls. Due to the variation of VOC emission within species, we regarded it to be 'presence' as long as one or more individual emitted the compound in a greater amount than in the air controls. The plant-animal interaction matrix as well as the plant-herbivore association matrix were constructed by pooling together the four plots sampled in our study. This was done in order to provide a better approximation to a community-wide pattern of communication.

We used the plant-VOC matrix with either 31 or 56 VOCs to test the robustness of our results to changes in the number of VOCs. In addition to the 20 target tree species that were used for the plant-VOC network in year 2018, we also randomly chose another 27 tree species in different succession stages and collected their VOCs. These 47 plants and their VOC patterns were used to test the robustness of our results to changes in species interaction networks from other years (2007-2017) (See bellow Section D Robustness tests concerning effects of sampling bias. Zu_etal_2020_Data_Archive.xlsx).

Note that in this study, we sampled plant VOCs in the field under natural conditions, reflecting an ecologically relevant chemical environment encountered by herbivores. Our data represent qualitative "snapshots" of VOCs likely including both constitute and induced VOCs. Additionally, we have taken a top-down perspective: if an herbivore can feed on a plant, it must be able to deal with the VOCs which the plant emits, despite the effect of each VOC on the herbivore (attract, 
deter, neutral). Because insects are known to have a large number of olfactory receptors with high sensitivity, it should therefore be reasonable to assume that the insects in our community take into account plant VOCs across spatial and temporal variation when deciding where to feed. However, future work can explore the capacity of our theoretical work in understanding chemical communication and species interactions by including different sets of VOCs (e.g., constitute and induced VOCs), VOCs from different organs (e.g., from flowers, leaves and roots), and different sets of partners across trophic levels (e.g., pollinators, microbes, parasitoids and predators). Moreover, experimental work could be further conducted to determine which VOCs are relevant for which herbivores. This includes measuring VOC profiles under controlled conditions, e.g., measuring before and after herbivore damage, conducting behavioral assays using synthetic compounds, using different VOC concentrations and combinations, and measuring from a larger number of samples per species. There are many such published studies which could perhaps be analyzed using the framework we propose here. Similar experiments can be conducted repeatedly along time (seasons) to explore temporal dynamics of chemical communication between plants and insects. 


\section{B Information theory}

For any binary $m \times n$ matrix, say object-signal $(O S)$ matrix with $m$ objects and $n$ signals and each element $o s_{i j}(i \in 1,2, \ldots, m, j \in 1,2, \ldots, n)$ representing the presence $o s_{i j}=1$ or absence $o s_{i j}=0$ of an interaction (or association), we can calculate the conditional entropy as $H_{n}(O \mid S)=$ $\sum_{j=1}^{n} P\left(S_{j}\right) H_{n}\left(O \mid S_{j}\right)$. Note that $H_{n}(O \mid S) \in(0,1)$, where the higher the value, the higher the uncertainty (information needed) to know the outcome (identity) of an object given that a specific signal was observed. In this calculation, $P\left(S_{j}\right)=\frac{\sum_{i=1}^{m} o s_{i, j}}{\sum_{i=1, j=1}^{m,} o s_{i, j}}$ corresponds to the probability of having signal $j$ given that signal was observed. Then, $H_{n}\left(O \mid S_{j}\right)=-\sum_{i=1}^{m} P\left(O_{i} \mid S_{j}\right) \log _{n} P\left(O_{i} \mid S_{j}\right)$, where $P\left(O_{i} \mid S_{j}\right)=\frac{o s_{i, j}}{\sum_{i=1}^{m} o s_{i, j}}$ corresponds to the probability of having object $i$ given that signal $j$ was observed.

We can also calculate the mutual information $I(O, S)=H_{m}(O)-H_{n}(O \mid S)$, where $H_{n}(O \mid S)$ is calculated as previously mentioned, and $H_{m}(O)=-\sum_{i=1}^{m} P\left(O_{i}\right) \log _{n} P\left(O_{i}\right)$. In our analysis, objects correspond to species and given that we generated qualitative matrices (with 0 s and $1 \mathrm{~s}$ ), we did not use species abundances and considered $P\left(O_{i}\right)=\frac{1}{m}$. Note that $I(O, S)=I(S, O) \in(0,1)$, where the higher the value, the greater the information (identity) that we can obtain about an object by knowing the presence or absence of a signal.

Similarly, to calculate the mutual information between an object and a library (combination) of signals, we constructed new matrices describing an object and a library (i.e., a $O L$ matrix). For example, for a given library size (say 2) with a given set of signals (say $S_{1}$ and $S_{2}$ ), all the unique combinations of the library (e.g., $(1,1),(1,0),(0,1)$, and $(0,0))$ would form the columns of the matrix and the objects form the rows. Then, we filled the elements of the $O L$ matrix with either 1 or 0 , depending on the presence or absence of the specific set of signals in the corresponding object. As the library size increases, the number of signal combinations exponentially increases, making potential analyses infeasible. For example, choosing a library size of 2 out of 50 will give 2, 450 different combinations, whereas choosing a size of 5 out of 50 will give 10, 593, 800 different combinations. Therefore, for each library size, we used 5000 unique random combinations and then calculated the maximum mutual information we could obtain from these combinations to represent the situation with a best set of VOCs. We calculated the mutual information between herbivores and VOCs as a function of the VOC library size based on the herbivore-VOC $(A V)$ matrix. Because the $A V$ matrix is weighted, we first scaled the number of VOCs for each herbivore based on the total number of plants that a given herbivore interacts with. This produced a probability matrix representing the chance that a herbivore associates with each of the VOCs. Next, we created 30 replicates or realizations of binary $A V$ matrices based on the corresponding probability for each interaction, mimicking the decoding process. Then, for each of these binary $A V$ matrices, we calculated the mutual information the same way as stated before for the $P V$ matrices. Then we calculated the median (due to the skewed distribution) mutual information based on these results from the 30 realizations. 


\section{Simulations and statistics}

To test the long-term behavior of the studied optimization mechanisms, we used three initial conditions for the $A P$ and $P V$ matrices. We defined the first initial condition by a matrix with highly specialized interactions. That is, each plant interacts with only one or maximum two (given the constraints imposed by the dimension of the matrices) herbivores and VOCs. The second initial condition was defined by a matrix with highly generalized interactions. That is, each plant interacts with all but one or maximum two herbivores and VOCs. The third initial condition was defined by randomly drawing values ( 1 or 0$)$ with different probabilities. Note that the third condition is the only one generating different matrices, hence, we explore many different randomization and probability values. To control for confounding factors related to dimensionality, all the simulated matrices had the the same dimension as the observed matrices.

For each initial condition (matrices), we calculated the conditional entropy (for $A P$ and $P V$ ) and the plant and animal fitness relationships $\left(\mathrm{F}_{P}=H(A \mid V)\right.$ and $\mathrm{F}_{A}=1-H(V \mid A)$, where $A V=A P \times P V)$. Then, the optimization mechanism began with herbivores, which were allowed to alter their interactions with plants (i.e., mutate 1 and 0 in the AP-matrix) to maximize their fitness relationships. Specifically, as shown in Figure 2, one element will mutate at each time, and a new fitness relationship will be calculated accordingly. The old matrix will remain if the new fitness relationship is smaller than the old value. Otherwise, the new matrix will fixate and will be used for the next mutation event. We assumed equal mutation rate for each link (plant-VOC link or herbivore-plant link) in the absence of any prior knowledge. Therefore, the number of random mutations that plants and herbivores can have for each round were proportional to the number of all possible links (i.e., number of elements in $P V$ and $A P$ matrices). However, different mutation rates for plants and herbivores mimicking unequal ability in combating in the arms-race can be further investigated. Here, the number of mutation events in herbivores is given by $20 \%$ of the total number of elements in the AP matrix. After this number of mutations, plants take a turn and maximize their fitness relationships by changing their association with VOCs (i.e., mutate elements in the $P V$ matrix in the same fashion as described before). The number of mutation events in plants is also given by $20 \%$ of the total number of elements in the $P V$ matrix. This alternated optimization scheme is repeated 1000 times. The number of mutations within each round does not change the pattern; it only changes the time to reach equilibrium (Fig. S1). Starting the optimization mechanism with plants or herbivores also does not affect the results.

To test whether the optimization mechanisms, starting from the simulated matrices, would converge to our field observations, we examined the long-term patterns of each simulated matrix. Specifically, we calculated conditional entropies and fitness relationships based on simulated matrices using the average values over the last 250 rounds of the simulated time series. The specific number of rounds did not change our results, but we used this number in order to provide a mean behavior over time. All the simulations and statistics were conducted with $\mathrm{R}$ version 3.5.2. (42). 


\section{Robustness tests concerning effects of sampling bias}

Sampling bias is an important concern in ecological studies (34). To test whether the sampling effort will affect our results, we conducted multiple paralleled analyses with different conditions: (1) community size, (2) number of VOCs, and (3) interaction network.

1. Community size: Will results be affected by a sub-sampling of the community? For this test, we used a subset of the plant-herbivore community (a specific plot with 8 plants and 16 herbivores instead of the entire pooled community as in the main text). We found that the results remain qualitatively equivalent regardless of community size, illustrating the scalability of our findings (Figure S2). We also performed simulations with many different sub-samples and they were all qualitatively equivalent to Figure S2.

2. Number of VOCs: Are our results affected by an incomplete plant VOC profile? Here, we were concerned about the potential effects of unidentified plant VOCs. For this test, we included 21 more undefined VOCs that were potential biogenic VOCs (see above Section Construction of field leaf scent collection and plant-VOC network under A Field data), and kept the interaction matrix unchanged. We found that the results still remain qualitatively equivalent (Figure S3, all VOC information see Zu_etal_2020_Data_Archive.xlsx).

3. Interaction network: Will results be affected by sampling the interaction network in a different place or year? To test this, we used information from previous years (from 2007-2017 using data from Ref. (44)and repeated all our analyses. Specifically, using the pool of plant species and VOC information collected in 2018, we checked for those plant species present in a given previous year together with all their feeding herbivores present in such year to form yearly interaction matrices. This made both the identity and number of plant-herbivore interactions to change each year. Note that here we assumed that VOCs of a specific plant species in a specific plot remain qualitatively unchanged through these years. We found that the results remain qualitatively equivalent regardless of the identity and number of species interactions (Figures S4-S6, all interaction networks under analyses from previous years see Zu_etal_2020_Data_Archive.xlsx).

The robustness of these results is aligned with previous work (45) that has found no change of global network measures in the data despite large beta diversity along each year (turnovers of herbivores). This result further indicates that a community can be formed by different species, but the interaction patterns (and likely the mechanism to drive the interaction patterns) do not change at our level of resolution. 

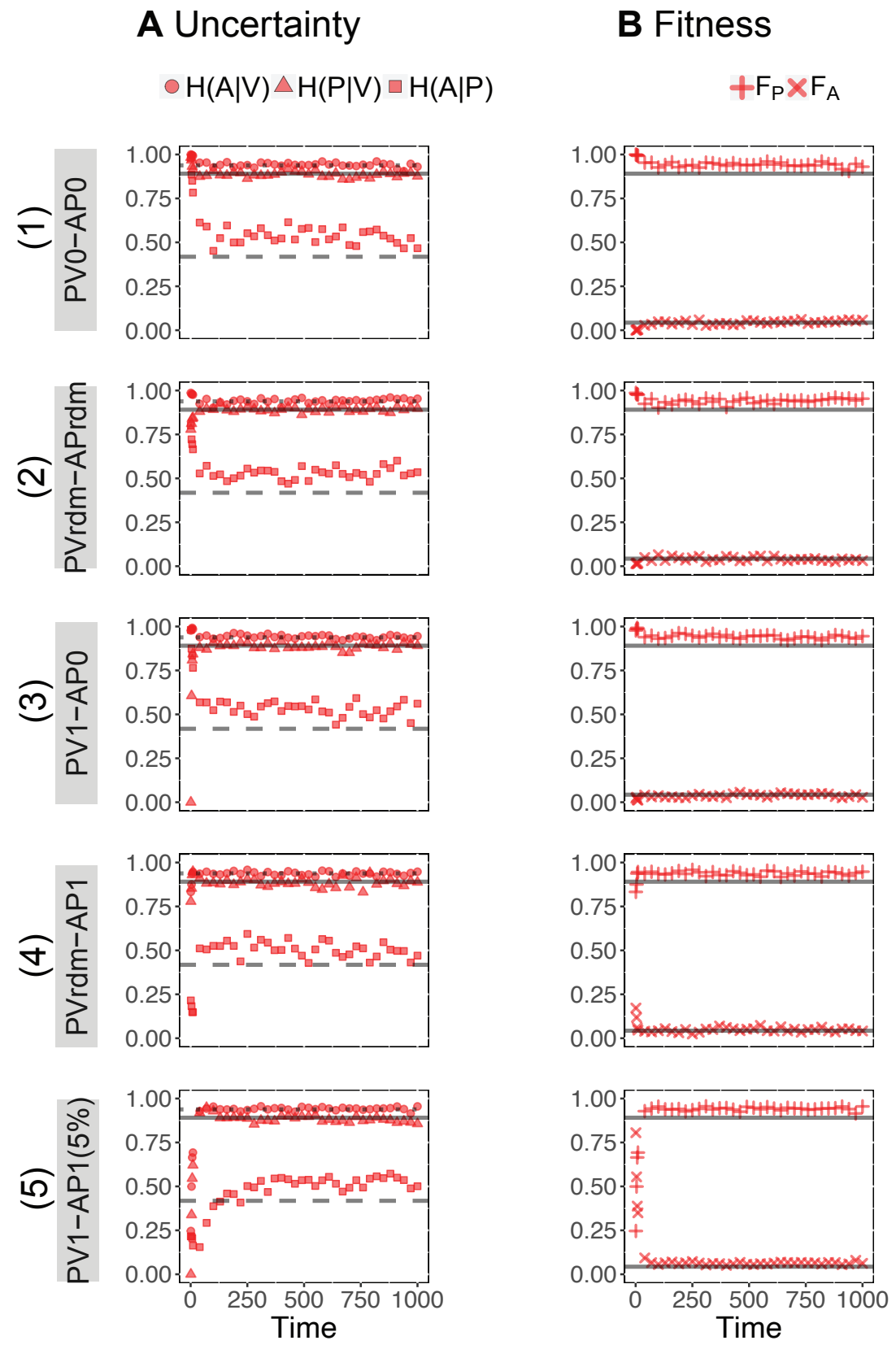

Figure S1: Simulations from different starting matrices. The simulations started from PV and AP matrices that are: (1) both generalized relationship matrices; (2) both random; (3) PV specialized and AP generalized; (4) PV random and AP specialized; (5) both specialized, and the element mutation rate is $5 \%$ of the total elements (instead of $20 \%$ for all the other cases). Panel A illustrates the uncertainty (conditional entropy) of the three matrices simulated over 1000 time steps derived from our proposed optimization mechanism. The circles, triangles and squares correspond to the conditional entropies $H(A \mid V), H(P \mid V)$ and $H(A \mid P)$, respectively. The solid, dotted and dashed lines stand for to the corresponding conditional entropies from field observations. For the same simulation, in Panel $\mathbf{B}$, the + and $\times$ symbols correspond to the fitness relationships of plants $\left(F_{P}\right)$ and herbivores $\left(F_{A}\right)$, respectively. The solid lines correspond to the field observations. 
(1) Field observation

A

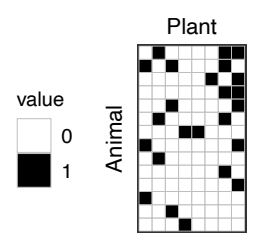

(2) Simulation results
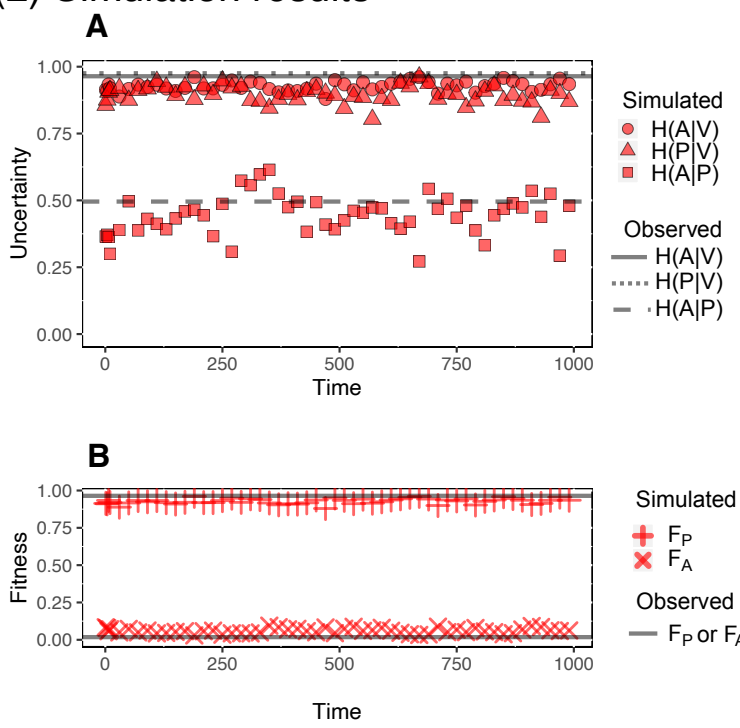

C

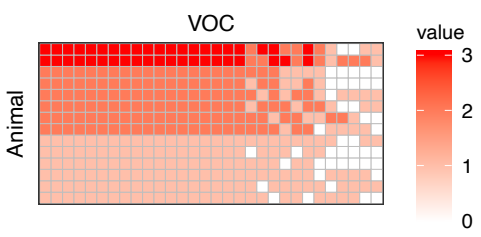

C

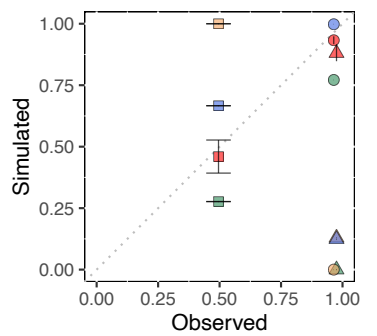

Optimization

mechanisms

- $\operatorname{Min}_{\mathrm{A}}-\operatorname{Max}_{\mathrm{P}} \quad$ - Field

- $\operatorname{Min}_{\mathrm{A}}-\operatorname{Min}_{\mathrm{P}}$

- $\operatorname{Max}_{A}-\operatorname{Min}_{P}$

- $\operatorname{Max}_{\mathrm{A}}-\operatorname{Max}_{\mathrm{P}}$

\section{(3) Emergence of cumulative mutual information}
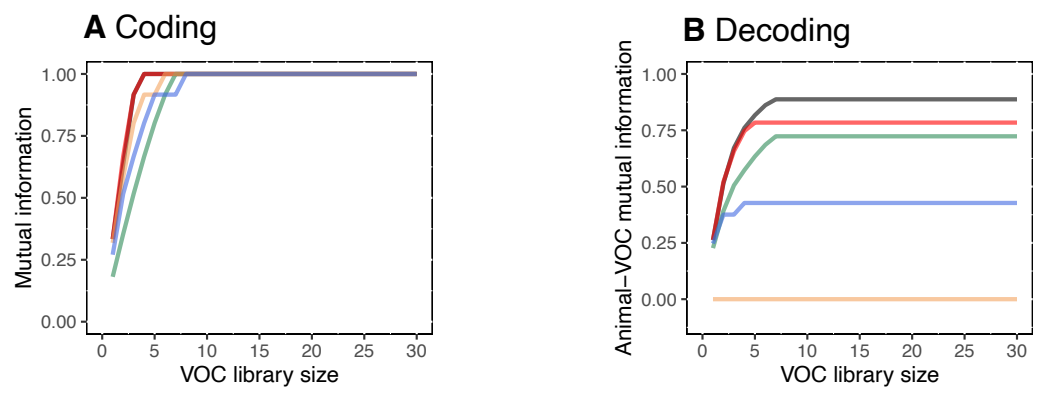

Figure S2: Test with a subset of the plant-herbivore community. This analysis corresponds to a subset of the community from year 2018. This includes 16 animal species, 8 plant species, and 30 VOCs. Here we have 30 VOCs instead of 31 because one VOC was emitted by none of the 8 plant species. For detailed figure captions see Figures 1, 3, and 4 in the main text. 


\section{(1) Field observation}
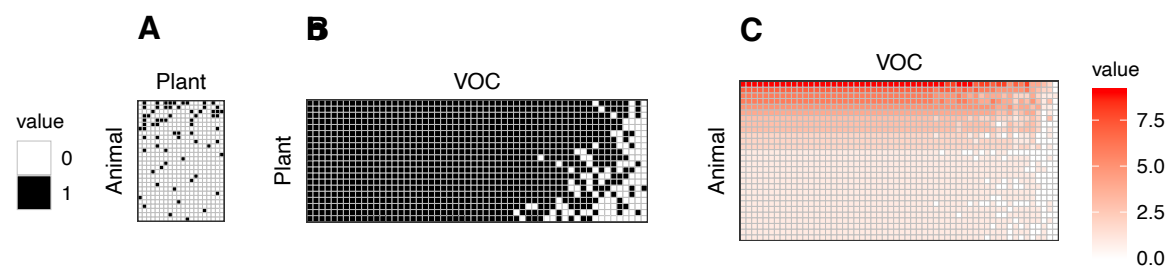

\section{(2) Simulation results}

A

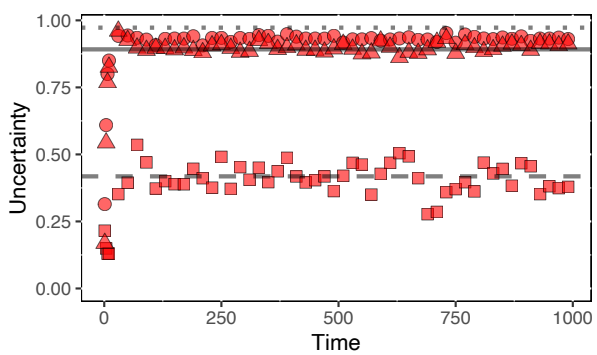

B

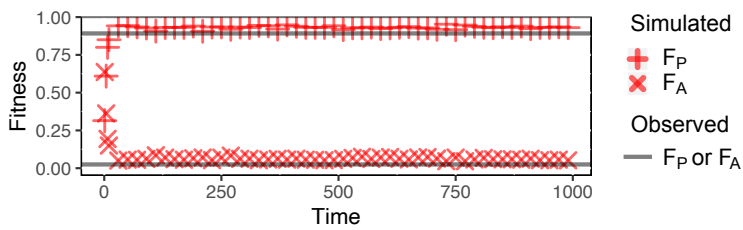

C

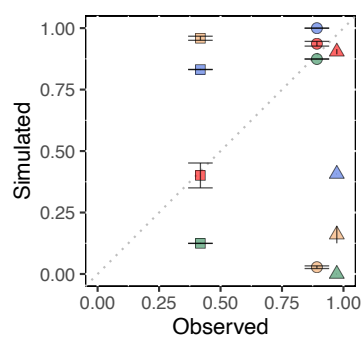

Optimization mechanisms

$-\operatorname{Min}_{A}-\operatorname{Max}_{P} \quad-$ Field

- $\operatorname{Min}_{\mathrm{A}}-\operatorname{Min}_{\mathrm{P}}$

- $\operatorname{Max}_{A}-\operatorname{Min}_{P}$

- $\operatorname{Max}_{A}-\operatorname{Max}_{P}$

\section{(3) Emergence of cumulative mutual information}
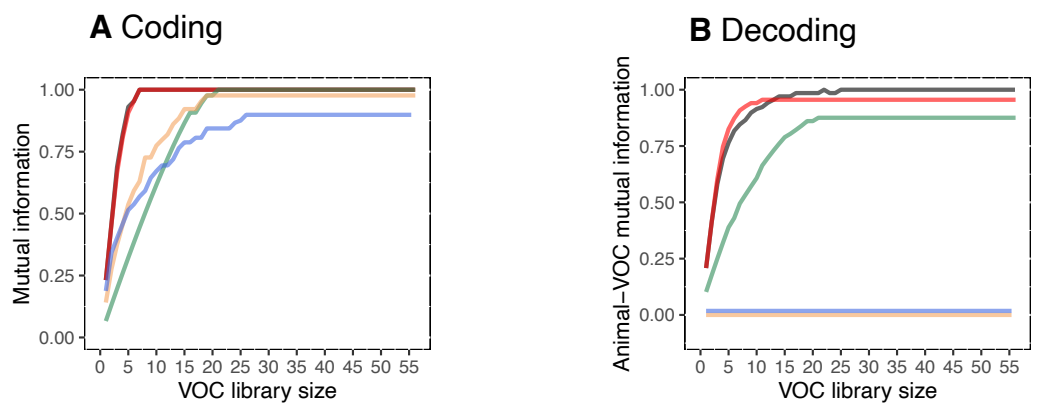

Figure S3: Test with 56 VOCs. Here we used 56 VOCs (instead of 31 VOCs as in the main text, which did not include biogenic VOCs). This corresponds to 28 animal species, 20 plant species, and 56 VOCs. For detailed figure captions see Figures 1, 3, and 4 in the main text. 
(1) Field observation
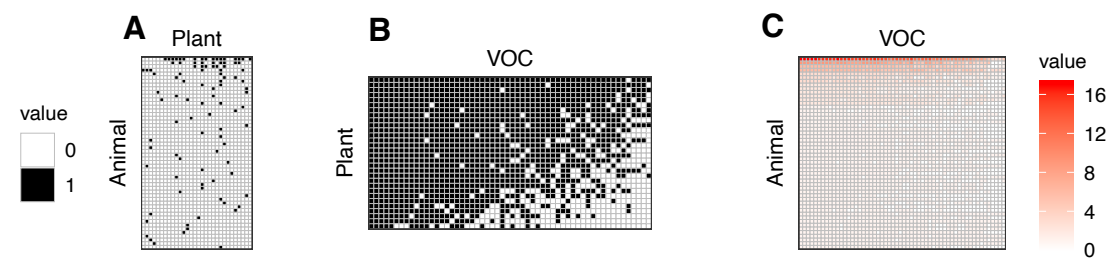

(2) Simulation results
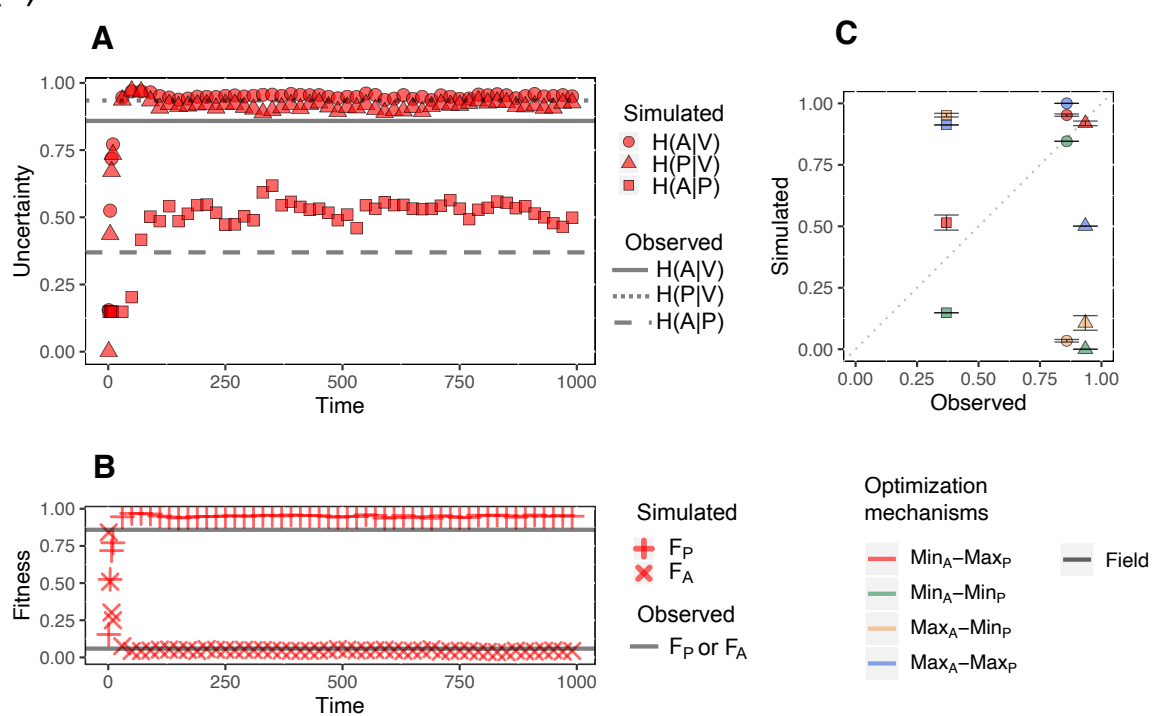

\section{(3) Emergence of cumulative mutual information}
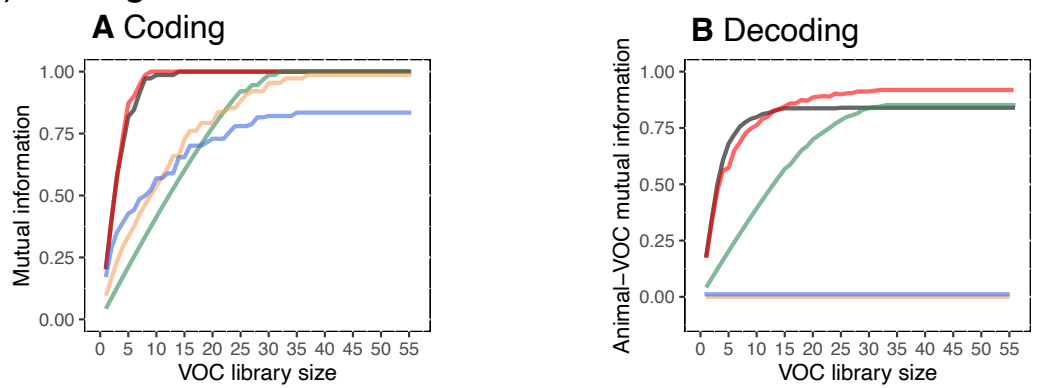

Figure S4: Test with different network communities - year 2017. We used the plantherbivore interaction matrix sampled in the year 2017. This corresponds to 52 animal species, 30 plant species, and 56 VOCs. For detailed figure captions see Figures 1, 3, and 4 in the main text. 
(1) Field observation
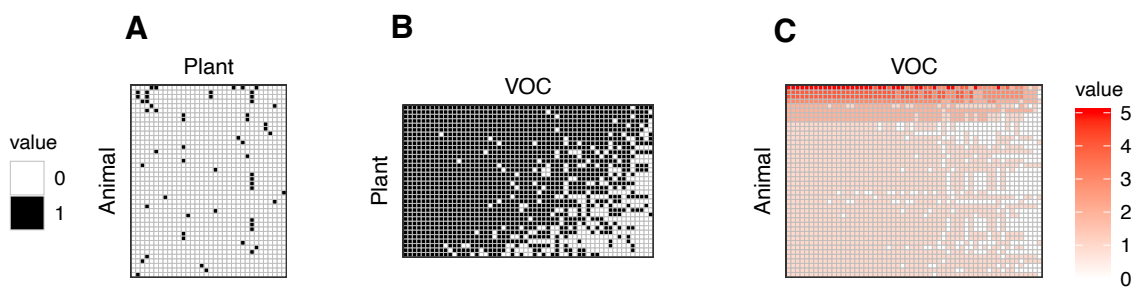

\section{(2) Simulation results}

A

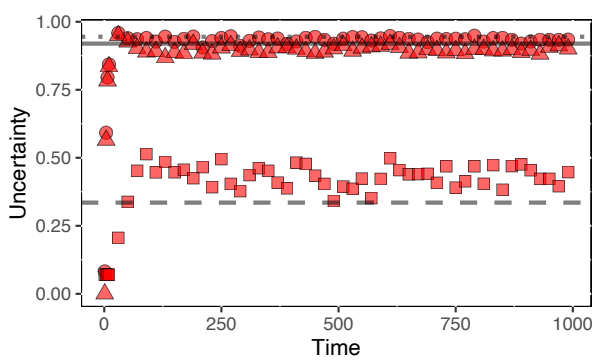

B

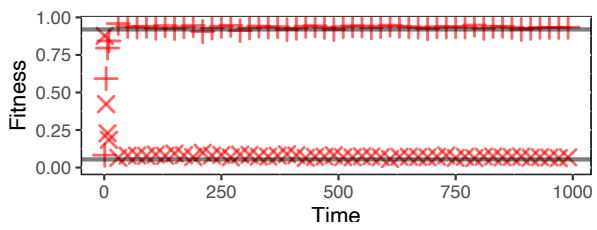

Simulated

$+F_{P}$

Observed

- $F_{P}$ or $F_{A}$
C

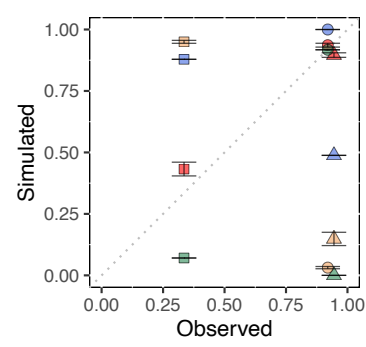

$$
\begin{aligned}
& \text { Optimization } \\
& \text { mechanisms } \\
& -\operatorname{Min}_{A}-\operatorname{Max}_{P} \quad \text { - Field } \\
& -\operatorname{Min}_{A}-M_{P} \\
& -\operatorname{Max}_{A}-\operatorname{Min}_{P} \\
& -\operatorname{Max}_{A}-\operatorname{Max}_{P}
\end{aligned}
$$

(3) Emergence of cumulative mutual information
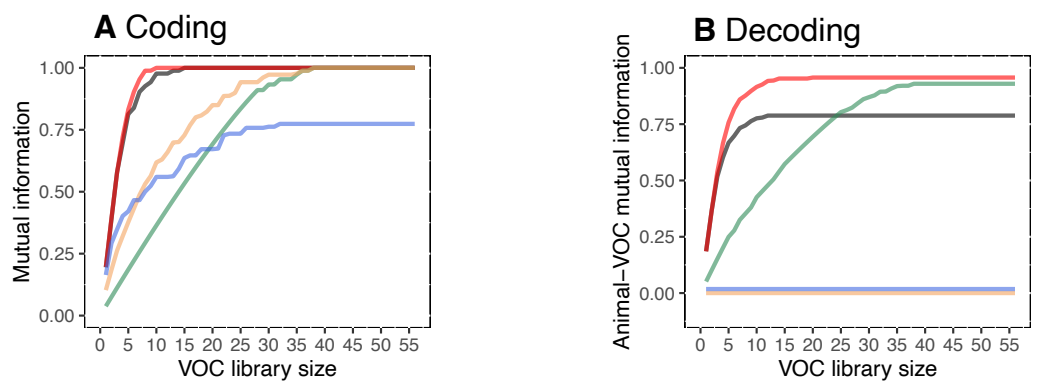

Figure S5: Test with different network communities - year 2007. We used the plantherbivore interaction matrix sampled in the year 2007. This corresponds to 42 animal species, 34 plant species, and 56 VOCs. For detailed figure captions see Figures 1, 3, 4 in the main text. 


\section{A Uncertainty}

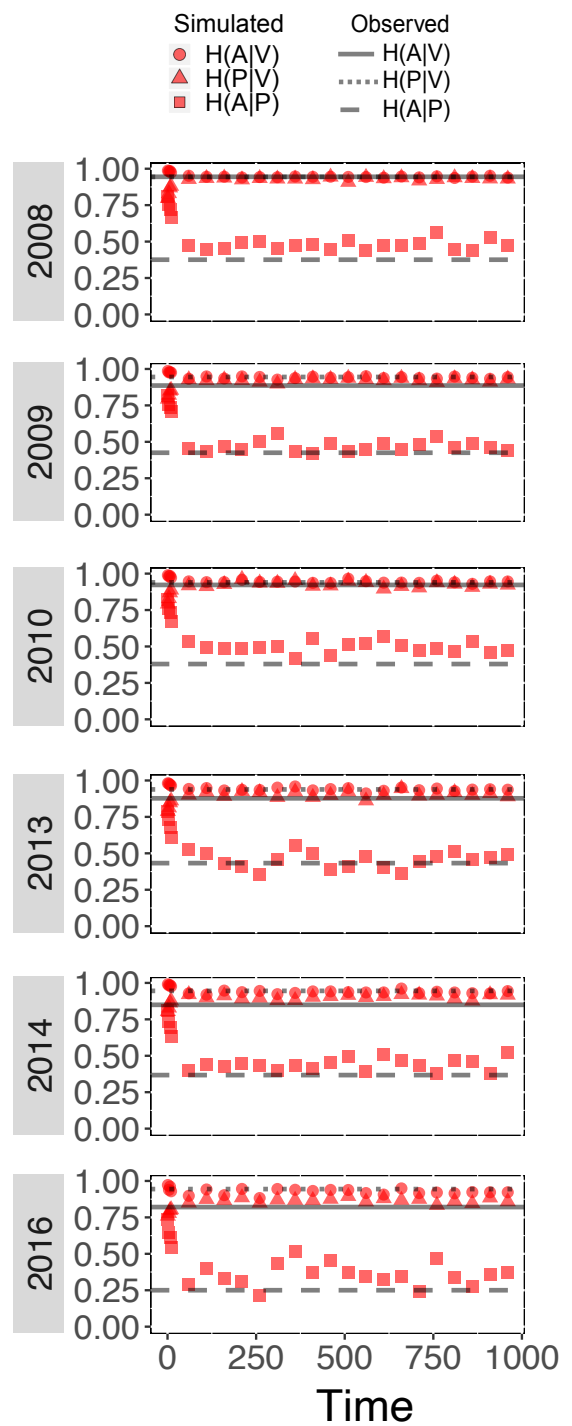

\section{B Fitness}

$$
\begin{array}{ll}
\text { Simulated } & \text { Observed } \\
+F_{\mathrm{P}} & -\mathrm{F}_{\mathrm{P}} \text { or } \mathrm{F}_{\mathrm{A}}
\end{array}
$$

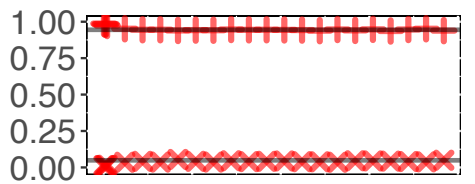

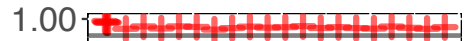

0.75

0.50

0.25

$0.00 \ldots$
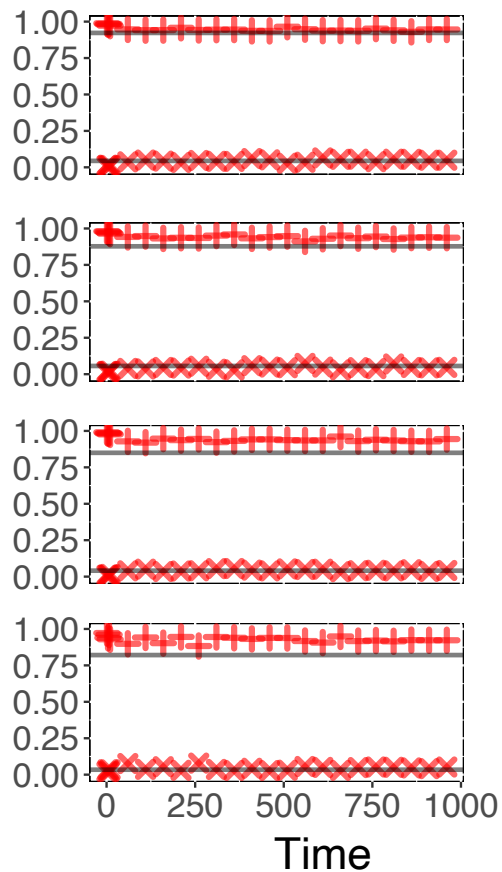

Figure S6: Summary test with different network communities - years 2008-2016. We used the plant-herbivore interaction matrix sampled in the years 2008-2016. The number of VOCs is 56 for all these years. Number of animal species (A) and number of plant species (P) in each year are: 2008 (75A-28P), 2009 (57A-25P), 2010 (63A-22P), 2013 (36A-20P), 2014 (37A-25P), 2016 (13A-14P). The year 2015 was not sampled due to bad weather conditions generated by Hurricane Patricia boege2019temporal. The years 2011 and 2012 are missing because no VOC information is available for the tree species sampled that year. For detailed figure captions see Figure 3 in the main text. 
Table S1: Herbivore-plant interaction network. Data for Figure 1A in the main text.

\begin{tabular}{|c|c|c|c|c|c|c|c|c|c|c|c|c|c|c|c|c|c|c|c|c|c|c|c|c|c|c|c|c|c|}
\hline & & 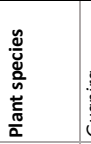 & 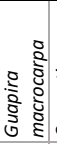 & 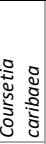 & 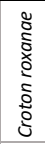 & 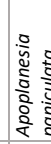 & 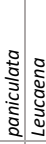 & 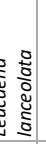 & 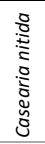 & 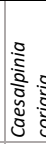 & & & & & & 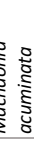 & t. & & & 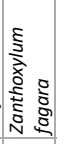 & 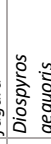 & & & & 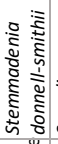 & 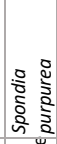 & 옹 & & \\
\hline & & 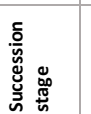 & 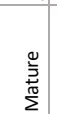 & 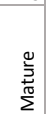 & 总 & & 惫 & 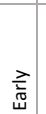 & 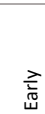 & 골 & & 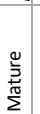 & 곯 & & 童 & 忞 & & ti & 䇊 & 颃 & 离 & & 离 & 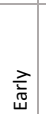 & 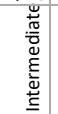 & 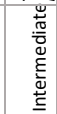 & 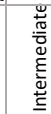 & & \\
\hline & & 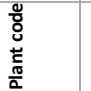 & $\sum_{0}^{\pi}$ & § & 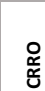 & & 这 & 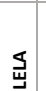 & $\bar{z}$ & 乌্ & & 髉 & $\frac{8}{a}$ & & 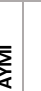 & $\frac{u}{\Sigma}$ & & $\frac{5}{5}$ & 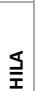 & $\frac{\mathbb{K}}{N}$ & 崖 & & 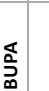 & 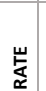 & 品 & àa & 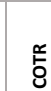 & & \\
\hline $\begin{array}{l}\text { Herbivore } \\
\text { code }\end{array}$ & \begin{tabular}{|l} 
Herbivore \\
species
\end{tabular} & & & & & & & & & & & & & & & & & & & & & & & & & & & Sun & \\
\hline 03 & Psychidae sp1. & & 1 & 0 & 1 & 1 & 1 & 1 & 0 & & 0 & 1 & 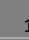 & & 0 & 0 & & 0 & 0 & 0 & & & 1 & 0 & 1 & 1 & & 0 & 9 \\
\hline 030 & Orgya sp. & & 0 & 0 & 1 & 1 & 1 & 1 & 0 & & 0 & 0 & 1 & & 0 & 0 & & 0 & 0 & 1 & 0 & 0 & 0 & 1 & 0 & 1 & 0 & 0 & 7 \\
\hline 018 & Hypercompe sp. & & 1 & 1 & 0 & 0 & 0 & 0 & 1 & c & 0 & 0 & 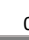 & & 1 & 0 & & 0 & 0 & 0 & 1 & 1 & 0 & 1 & 0 & 0 & 0 & 0 & 6 \\
\hline 014 & Psychidae sp2. & & 1 & 0 & 0 & 0 & 1 & 0 & 0 & & 0 & 0 & 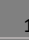 & & 0 & 1 & & 1 & 0 & 0 & 0 & 0 & 0 & 1 & 0 & 0 & 0 & 0 & 6 \\
\hline 0347 & Eudesmia menea & & 0 & 0 & & 0 & 0 & 1 & 1 & & 0 & 0 & 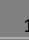 & & 0 & 0 & & 0 & 0 & 0 & 0 & 0 & 0 & 0 & 0 & 1 & 1 & 1 & 5 \\
\hline 024 & Wockia chewbac & & 0 & 0 & 0 & 0 & 0 & 1 & 1 & & 0 & 0 & 1 & & 0 & 0 & & 0 & 0 & 0 & 1 & 1 & 0 & 0 & 0 & 0 & 0 & 0 & 4 \\
\hline 0741 & Lepidoptera sp1 & & 0 & 0 & 0 & 0 & 0 & 0 & 0 & & 0 & 0 & 1 & & 0 & 0 & & 0 & 1 & 0 & 0 & 0 & 0 & 1 & 0 & 0 & 0 & 0 & 3 \\
\hline 044 & Misoria amra & & 0 & 0 & 0 & 0 & 0 & 0 & 1 & & 0 & 0 & $c$ & & 0 & 0 & & 0 & 0 & 1 & 0 & 0 & 1 & 0 & 0 & 0 & 0 & 0 & 3 \\
\hline 0161 & Dalcerides sp1. & & 0 & 0 & 0 & 0 & 0 & 1 & 0 & & 0 & 0 & 1 & & 0 & 0 & & 0 & 0 & 1 & 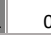 & 0 & 0 & 0 & 0 & 0 & 0 & 0 & 3 \\
\hline 069 & Diaphania jairus & & 0 & 0 & 0 & 0 & 0 & 0 & 0 & & 0 & 0 & & & 0 & 0 & & 1 & 0 & 0 & 0 & 0 & 0 & 1 & 0 & 0 & & 0 & 2 \\
\hline 0752 & Lepidoptera $\mathrm{sp2}$ & & 0 & 0 & 0 & 0 & 0 & 1 & 0 & & 1 & 0 & & & 0 & 0 & & 0 & 0 & 0 & 0 & 0 & 0 & 0 & 0 & 0 & 0 & 0 & 2 \\
\hline 0718 & Erebinae sp1. & & 0 & 0 & 0 & 0 & 0 & 0 & 0 & & 0 & 0 & 1 & & 0 & 0 & & 0 & 0 & 0 & 0 & 0 & 0 & 0 & 0 & 1 & 0 & 0 & 2 \\
\hline 0765 & Lepidoptera sp4 & & 0 & 1 & 0 & 0 & 0 & 0 & 0 & & 0 & 0 & & & 0 & 0 & & 0 & 0 & 0 & 0 & 0 & 0 & 0 & 0 & 0 & 0 & 0 & 1 \\
\hline 0719 & Erebinae sp2. & & 0 & 0 & 0 & 0 & 0 & 0 & 0 & & 0 & 0 & c & & 0 & 0 & & 0 & 0 & 0 & 0 & 0 & 0 & 0 & 1 & 0 & 0 & 0 & 1 \\
\hline 0230 & Anacamptodes h & herse & 0 & 0 & 0 & 0 & 0 & 0 & 0 & & 0 & 0 & & & 0 & 0 & & 0 & 0 & 0 & 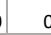 & 0 & 0 & 0 & 0 & 1 & 0 & 0 & 1 \\
\hline 0190 & Cargida pyrrha & & 0 & 0 & 0 & 0 & 0 & 0 & 0 & & 0 & 0 & & & 0 & 0 & & 0 & 0 & 0 & 0 & 0 & 0 & 0 & 0 & 0 & 1 & 1 & 1 \\
\hline 0775 & Lepidoptera sp3 & & 0 & 0 & 0 & 0 & 0 & 0 & 0 & & 0 & 0 & & & 0 & 0 & & 0 & 0 & 0 & 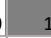 & 1 & 0 & 0 & 0 & 0 & 0 & 0 & 1 \\
\hline 052 & Gonodonta pyrg & & 0 & 0 & 0 & 0 & 0 & 0 & 0 & & 0 & 0 & & & 0 & 0 & & 1 & 0 & 0 & 0 & 0 & 0 & 0 & 0 & 0 & & 0 & 1 \\
\hline 025 & Michaelophorus & & 0 & 0 & 0 & 0 & 0 & 0 & 1 & & 0 & 0 & & & 0 & 0 & & 0 & 0 & 0 & 0 & 0 & 0 & 0 & 0 & 0 & & 0 & 1 \\
\hline 01 & Dalcerides sp2. & & 0 & 0 & & 0 & 0 & 1 & 0 & & 0 & 0 & & & 0 & 0 & & 0 & 0 & 0 & 0 & 0 & 0 & 0 & 0 & 0 & 0 & 0 & 1 \\
\hline 071 & Helia sueroides & & 0 & 0 & & 0 & 0 & 0 & 0 & & 0 & 0 & & & 0 & 0 & & 0 & 0 & 0 & 0 & 0 & 1 & 0 & 0 & 0 & & 0 & 1 \\
\hline 0768 & Deinopa biligula & & 0 & 0 & & 0 & 0 & 0 & 0 & & 0 & 0 & & & 0 & 0 & & 0 & 0 & 0 & 0 & 0 & 0 & 1 & 0 & 0 & & 0 & 1 \\
\hline 0341 & Perigonia ilus & & 0 & 0 & & 0 & 0 & 0 & 0 & & 0 & 0 & & & 0 & 0 & & 0 & 1 & 0 & , & 0 & 0 & 0 & 0 & 0 & 0 & 0 & 1 \\
\hline 0114 & Lophocampa citr & & 0 & 0 & & 0 & 0 & 0 & 0 & & 0 & 0 & c & & 0 & 0 & & 0 & 0 & 1 & 0 & 0 & 0 & 0 & 0 & 0 & 0 & 0 & 1 \\
\hline 0213 & Melipotis perper & ndicularis & 0 & 0 & & 0 & 0 & 0 & 0 & & 0 & 0 & & & 0 & 0 & & 0 & 0 & 0 & 0 & 0 & 0 & 0 & 0 & 0 & & 0 & 1 \\
\hline 0746 & Geometridae sp & & 0 & 0 & 1 & 1 & 0 & 0 & 0 & & 0 & 0 & & & 0 & 0 & & 0 & 0 & 0 & 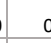 & 0 & 0 & 0 & 0 & 0 & 0 & 0 & 1 \\
\hline 0700 & Noctuidae sp1. & & 0 & 0 & 0 & 0 & 1 & 0 & 0 & & 0 & 0 & & & 0 & 0 & & 0 & 0 & 0 & 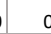 & 0 & 0 & 0 & 0 & 0 & 0 & 0 & 1 \\
\hline 096 & Syllepis hortalis & & 0 & 0 & & 0 & 0 & 0 & 0 & & 0 & 1 & & & 0 & 0 & & 0 & 0 & 0 & 0 & 0 & 0 & 0 & 0 & 0 & 0 & 0 & 1 \\
\hline
\end{tabular}


Table S2: VOC-plant association matrix. Data used for the transposed PV matrix in Figure 1B in the main text.

\begin{tabular}{|c|c|c|c|c|c|c|c|c|c|c|c|c|c|c|c|c|c|c|c|c|c|c|c|c|c|}
\hline & & & & & \multicolumn{20}{|c|}{ Plant code } & \\
\hline & & & & & 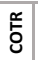 & $\frac{2}{a}$ & 号 & $\underset{⿱ 亠 凶}{\infty}$ & 矛 & 宮 & 岕 & 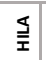 & $\frac{5}{i}$ & $\frac{u}{4}$ & $\sum_{\Sigma}^{\bar{\alpha}}$ & $\frac{\mathrm{U}}{2}$ & 至 & ষ্ড & $\bar{z}$ & $\underset{\exists}{\Psi}$ & $\begin{array}{ll} \\
\frac{a}{\alpha} \\
\frac{0}{\alpha}\end{array}$ & 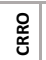 & ర్త & 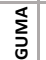 & \\
\hline ID & PCgroup & $\begin{array}{l}\text { VOC name } \\
\text { (NIST17_library) }\end{array}$ & $\begin{array}{l}\mathrm{m} / \mathrm{z} \\
\text { used }\end{array}$ & $\begin{array}{c}\text { Retention } \\
\text { time } \\
\text { (minute) }\end{array}$ & & & & & & & & & & & & & & & & & & & & & Sum \\
\hline 1 & $\mathrm{x} 112$ & (Z)-3-Hexenol & 67.10 & 10.86 & 1 & 1 & 1 & 1 & 1 & 1 & 1 & 1 & 1 & 1 & $\overline{1}$ & 1 & 1 & 1 & 1 & 1 & 1 & $\overline{1}$ & 1 & 1 & 20 \\
\hline 2 & X250 & (E)-2-Hexenal & 39.10 & 10.79 & 1 & 1 & 1 & 1 & 1 & 1 & 1 & 1 & 1 & 1 & 1 & 1 & 1 & 1 & 1 & 1 & 1 & 1 & 1 & 1 & 20 \\
\hline 3 & X381 & $\begin{array}{l}\text { Butanoic acid, 3- } \\
\text { methyl-, 3- } \\
\text { methylbutyl ester }\end{array}$ & 103.10 & 19.71 & 1 & 1 & 1 & 1 & 1 & 1 & 1 & 1 & 1 & 1 & 1 & 1 & 1 & 1 & 1 & 1 & 1 & 1 & 1 & 1 & 20 \\
\hline 4 & X406 & $\begin{array}{l}\text { 2-Methylbutanal } \\
\text { oxime }\end{array}$ & 86.10 & 10.82 & 1 & 1 & 1 & 1 & 1 & 1 & 1 & 1 & 1 & 1 & 1 & 1 & 1 & 1 & 1 & 1 & 1 & 1 & 1 & 1 & 20 \\
\hline 5 & X814 & 2-Bromododecane & 170.20 & 25.98 & 1 & 1 & 1 & 1 & 1 & 1 & 1 & 1 & 1 & 1 & 1 & 1 & 1 & 1 & 1 & 1 & 1 & 1 & 1 & 1 & 20 \\
\hline 6 & X892 & 3-Heptanone & 114.20 & 11.94 & 1 & 1 & 1 & 1 & 1 & 1 & 1 & 1 & 1 & 1 & 1 & 1 & 1 & 1 & 1 & 1 & 1 & 1 & 1 & 1 & 20 \\
\hline 7 & $\mathrm{X} 438$ & (Z)-Linalool oxide & 94.10 & 18.57 & 1 & 1 & 1 & 1 & 1 & 1 & 1 & 1 & 0 & 1 & 1 & 1 & 1 & 1 & 1 & 1 & 1 & 1 & 1 & 1 & 19 \\
\hline 8 & X6 & (Z)- $\beta$-Ocimene & 93.10 & 17.69 & 1 & 1 & 1 & 1 & 1 & 1 & 1 & 1 & 1 & 1 & 0 & 1 & 1 & 1 & 1 & 1 & 1 & 1 & 1 & 1 & 19 \\
\hline 9 & X651 & Eucalyptol & 139.20 & 17.29 & 1 & 1 & 1 & 1 & 1 & 1 & 0 & 1 & 1 & 1 & 1 & 1 & 1 & 1 & 1 & 1 & 1 & 1 & 1 & 1 & 19 \\
\hline 10 & X1735 & Isoledene & 204.20 & 29.70 & 1 & 1 & 1 & 1 & 1 & 1 & 1 & 1 & 1 & 1 & 1 & 1 & 1 & 1 & 1 & 1 & 1 & 1 & 0 & 1 & 19 \\
\hline 11 & X1789 & Pyridine & 79.10 & 7.29 & 1 & 1 & 1 & 1 & 1 & 1 & 1 & 1 & 1 & 1 & 1 & 1 & 1 & 1 & 1 & 1 & 1 & 1 & 1 & 0 & 19 \\
\hline 12 & X403 & Butylbutyrate & 89.10 & 15.96 & 1 & 1 & 1 & 1 & 1 & 1 & 1 & 1 & 1 & 1 & 1 & 1 & 1 & 1 & 1 & 1 & 1 & 1 & 1 & 0 & 19 \\
\hline 13 & X168 & Benzaldehyde & 77.10 & 14.84 & 1 & 1 & 1 & 1 & 1 & 1 & 1 & 1 & 1 & 1 & 1 & 0 & 1 & 1 & 1 & 1 & 1 & 1 & 1 & 0 & 18 \\
\hline 14 & X825 & Heptanal & 70.10 & 12.44 & 1 & 1 & 1 & 1 & 1 & 1 & 1 & 1 & 1 & 1 & 1 & 1 & 1 & 1 & 0 & 1 & 1 & 1 & 1 & 0 & 18 \\
\hline 15 & X961 & Humulene & 77.10 & 29.78 & 1 & 1 & 1 & 1 & 1 & 1 & 1 & 1 & 1 & 1 & 1 & 1 & 1 & 1 & 1 & 1 & 1 & 1 & 0 & 0 & 18 \\
\hline 16 & X14 & $\begin{array}{l}\text { (E)-4,8-Dimethylnona- } \\
\text { 1,3,7-triene (DMNT) }\end{array}$ & 69.10 & 19.90 & 1 & 1 & 1 & 1 & 1 & 1 & 1 & 1 & 0 & 1 & 1 & 1 & 1 & 0 & 1 & 1 & 1 & 0 & 1 & 1 & 17 \\
\hline 17 & X223 & 2,3-Butanediol & 45.10 & 8.55 & 1 & 1 & 1 & 0 & 1 & 1 & 1 & 0 & 1 & 1 & 1 & 1 & 1 & 1 & 1 & 1 & 1 & 1 & 1 & 0 & 17 \\
\hline 18 & $\mathrm{X} 40$ & $\alpha$-Copaene & 119.10 & 27.72 & 1 & 1 & 1 & 1 & 1 & 1 & 1 & 1 & 1 & 1 & 1 & 1 & 1 & 1 & 1 & 1 & 1 & 0 & 0 & 0 & 17 \\
\hline 19 & X105 & $\begin{array}{l}\text { Methoxyphenyl } \\
\text { Oxime }\end{array}$ & 133.00 & 12.55 & 1 & 0 & 0 & 1 & 1 & 1 & 1 & 1 & 1 & 1 & 0 & 1 & 1 & 1 & 1 & 1 & 1 & 0 & 1 & 1 & 16 \\
\hline 20 & X516 & $\begin{array}{l}\text { 2,3-Dimethyl-2-(1- } \\
\text { methylethyl)- } \\
\text { butanoic acid }\end{array}$ & 116.10 & 21.82 & 1 & 1 & 1 & 1 & 1 & 1 & 1 & 1 & 1 & 1 & 1 & 1 & 1 & 1 & 1 & 1 & 0 & 0 & 0 & 0 & 16 \\
\hline 21 & X265 & $\begin{array}{l}\text { 1,2,3-Trimethyl- } \\
\text { cyclopent-2- } \\
\text { enecarboxaldehyde }\end{array}$ & 109.10 & 16.25 & 1 & 1 & 1 & 1 & 1 & 1 & 1 & 1 & 1 & 1 & 0 & 1 & 0 & 1 & 1 & 1 & 0 & 0 & 0 & 1 & 15 \\
\hline 22 & X774 & 1-Methyl-1H-pyrrole & 80.10 & 6.64 & 1 & 1 & 1 & 1 & 1 & 1 & 1 & 1 & 1 & 1 & 0 & 1 & 0 & 1 & 1 & 1 & 0 & 1 & 0 & 0 & 15 \\
\hline 23 & X4 & 2-Ethyl-1-hexanol & 56.10 & 17.12 & 1 & 1 & 1 & 0 & 1 & 1 & 1 & 1 & 1 & 1 & 0 & 1 & 1 & 1 & 1 & 0 & 0 & 1 & 0 & 0 & 14 \\
\hline 24 & X317 & $\alpha$-Pinene & 93.10 & 13.68 & 1 & 1 & 1 & 1 & 0 & 1 & 1 & 1 & 1 & 0 & 1 & 1 & 1 & 1 & 1 & 0 & 0 & 0 & 0 & 0 & 13 \\
\hline 25 & $\mathrm{X} 456$ & Hexanal & 40.00 & 8.76 & 1 & 1 & 1 & 1 & 1 & 1 & 1 & 0 & 1 & 1 & 1 & 1 & 0 & 0 & 0 & 1 & 1 & 0 & 0 & 0 & 13 \\
\hline 26 & X1898 & 2-Pentylfuran & 138.10 & 15.75 & 1 & 1 & 1 & 1 & 1 & 0 & 1 & 1 & 1 & 1 & 1 & 1 & 1 & 0 & 0 & 0 & 0 & 0 & 0 & 0 & 12 \\
\hline 27 & X196 & $\begin{array}{l}\text { 4,7-Dimethyl-- } \\
\text { undecane, }\end{array}$ & 44.10 & 18.12 & 1 & 1 & 1 & 0 & 1 & 0 & 1 & 0 & 1 & 0 & 1 & 0 & 1 & 0 & 0 & 0 & 1 & 1 & 1 & 0 & 11 \\
\hline 28 & X495 & Furfural & 96.00 & 10.08 & 1 & 1 & 1 & 1 & 1 & 1 & 1 & 0 & 0 & 0 & 1 & 0 & 1 & 0 & 0 & 0 & 1 & 1 & 0 & 0 & 11 \\
\hline 29 & X234 & (E)- $\beta$-Ocimene & 103.10 & 17.42 & 0 & 1 & 1 & 1 & 1 & 1 & 0 & 1 & 1 & 1 & 1 & 0 & 0 & 0 & 0 & 0 & 0 & 1 & 0 & 0 & 10 \\
\hline 30 & X1396 & Nonanoic acid & 74.10 & 25.11 & 1 & 1 & 1 & 1 & 0 & 1 & 1 & 1 & 0 & 0 & 1 & 0 & 0 & 0 & 0 & 0 & 0 & 0 & 0 & 0 & 8 \\
\hline 31 & X450 & $\begin{array}{l}2,4,6- \\
\text { Trihydroxybenzaldehy } \\
\text { de }\end{array}$ & 154.10 & 27.88 & 1 & 1 & 0 & 1 & 0 & 0 & 0 & 1 & 0 & 0 & 1 & 0 & 0 & 0 & 0 & 0 & 0 & 0 & 0 & 0 & 5 \\
\hline
\end{tabular}

